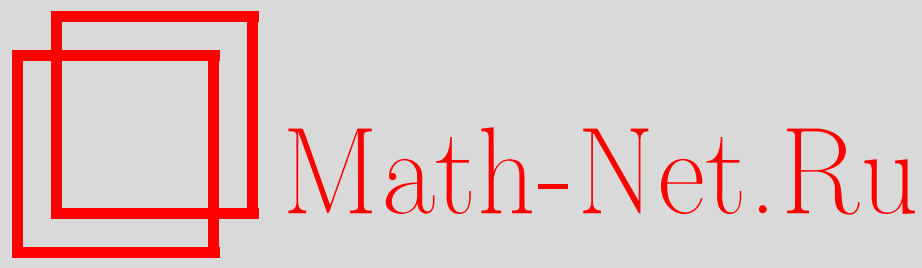

В. В. Жаринов, Законы сохранения, дифференциальные тождества и связи уравнений в частных производных, TMФ, 2015, том 185, номер 2, 227-251

DOI: https://doi.org/10.4213/tmf8960

Использование Общероссийского математического портала Math-Net.Ru подразумевает, что вы прочитали и согласны с пользовательским соглашением http://www . mathnet.ru/rus/agreement

Параметры загрузки:

IP : 3.81 .55 .215

26 апреля 2023 г., $15: 27: 34$

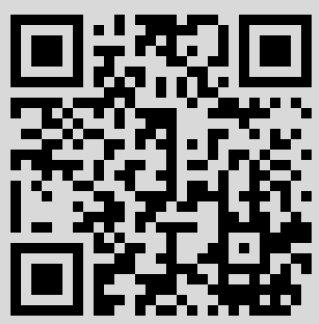




\title{
ЗАКОНЫ СОХРАНЕНИЯ, ДИФФЕРЕНЦИАЛЬНЫЕ ТОЖДЕСТВА И СВЯЗИ УРАВНЕНИЙ В ЧАСТНЫХ ПРОИЗВОДНЫХ
}

\begin{abstract}
Рассматриваются специфические когомологические свойства систем уравнений в частных производных, такие как маломерные законы сохранения и дифференциальные тождества. Показано, что такие свойства присущи сложным системам типа эволюционных систем со связями. Используемый здесь математический аппарат - алгебраический анализ систем уравнений в частных производных и когомологии дифференциальных алгебр и модулей.
\end{abstract}

Ключевые слова: дифференциальная алгебра, законы сохранения, дифференциальные тождества, дифференциальные связи.

DOI: $10.4213 / \operatorname{tmf} 8960$

\section{1. ВВЕДЕНИЕ}

Эта во многом обзорная статья основана на работах автора, опубликованных в течение последних тридцати лет [1]-[7]. В ней рассматриваются специфические свойства систем уравнений в частных производных, такие как маломерные законы сохранения и дифференциальные тождества. Оказывается, что такие свойства присущи системам неэволюционного типа. Мы называем систему уравнений в частных производных системой эволюиионного типа, если после подходящей замены переменных она записывается как эволюционная система, и системой неэволюиионного типа в противном случае.

В разделе 2 мы определяем все необходимые алгебраические ингредиенты (дифференциальные алгебры, дифференциальные идеалы, дифференциальные модули, дифференциальные многочлены, дифференциальные комплексы) и приводим необходимые когомологические утверждения (формула Грина, точность некоторых важных последовательностей).

Исследование выполнено за счет гранта Российского научного фонда (проект № 14-50-00005).

* Математический институт им. В.А. Стеклова Российской академии наук, Москва, Россия. E-mail: zharinov@mi.ras.ru 
В разделе 3 мы описываем системы уравнений в частных производных общего вида в алгебраическом подходе, рассматриваем основные элементы этого подхода (многообразие решений, дифференциальная алгебра, дифференциальный идеал, дифференциальный комплекс, маломерные законы сохранения и дифференциальные тождества, ассоциированные с данной системой уравнений в частных производных) и устанавливаем связь между пространствами законов сохранения и дифференциальных тождеств.

В разделе 4 мы показываем, что эволюционные системы не обладают маломерными законами сохранения, и изучаем эволюционные системы со связями (которые имеют неэволюционный тип). Оказывается, наличие маломерных законов сохранения у таких систем определяется когомологическими свойствами связей. Основные результаты здесь - теоремы 10, 11 и 12. Далее мы рассматриваем системы уравнений неразрывности и вычисляем их законы сохранения (теорема 13). Опираясь на этот результат, мы находим законы сохранения эволюционных систем со связями в виде нулевой дивергенции. В частности, мы показываем, что такие системы действительно имеют маломерные законы сохранения (теорема 14).

Общие результаты иллюстрируются рядом наглядных примеров, включая уравнения Янга-Миллса и Максвелла.

\section{2. ПРЕДВАРИТЕЛЬНЫЕ СВЕДЕНИЯ}

2.1. Обозначения. Мы используем стандартные обозначения:

- $\mathbb{R}, \mathbb{C}$ - поля вещественных и комплексных чисел;

- $\mathbb{Z}, \mathbb{Z}_{+}, \mathbb{N}$ - множества всех целых, неотрицательных целых, натуральных (положительных целых) чисел;

- $\mathbb{I}=\mathbb{Z}_{+}^{m}=\left\{i=\left(i^{1}, \ldots, i^{m}\right) \mid i^{\mu} \in \mathbb{Z}_{+}, 1 \leqslant \mu \leqslant m\right\}, m \in \mathbb{N},-$ аддитивный абелев моноид всех мультииндексов с покомпонентными операциями: $\left(i^{1}, \ldots, i^{m}\right)+$ $\left(j^{1}, \ldots, j^{m}\right)=\left(i^{1}+j^{1}, \ldots, i^{m}+j^{m}\right)$

- специальные мультииндексы: $0=(0, \ldots, 0),(\mu)=(0, \ldots, 0,1,0, \ldots, 0)$, где 1 стоит на $\mu$-м месте, $1 \leqslant \mu \leqslant m$;

- $|i|=i^{1}+\cdots+i^{m}$ для любого $i=\left(i^{1}, \ldots, i^{m}\right) \in \mathbb{I}$.

Далее,

- $\mathrm{X}=\mathbb{R}^{m}=\left\{x=\left(x^{1}, \ldots, x^{m}\right) \mid x^{\mu} \in \mathbb{R}, 1 \leqslant \mu \leqslant m\right\}, m \in \mathbb{N}$, - пространство независимых переменных;

- $\mathrm{U}=\mathbb{R}^{\mathrm{A}}=\left\{u=\left(u^{\alpha}\right) \mid u^{\alpha} \in \mathbb{R}, \alpha \in \mathrm{A}\right\}$ - пространство зависимых переменных, где $\mathrm{A}$ - некоторое множество индексов;

- $\mathbf{U}=\mathbb{R}_{\mathbb{I}}^{\mathrm{A}}=\left\{\mathbf{u}=\left(u_{i}^{\alpha}\right) \mid u_{i}^{\alpha} \in \mathbb{R}, \alpha \in \mathrm{A}, i \in \mathbb{I}\right\}-$ пространство дифференциальных переменных, проекция $\pi: \mathbf{U} \rightarrow \mathbf{U}, \mathbf{u}=\left(u_{i}^{\alpha}\right) \mapsto u=\left(u^{\alpha}\right)$, где $u^{\alpha}=u_{0}^{\alpha}$;

- $\mathbf{X} \mathbf{U}=\mathbf{X} \times \mathbf{U}$ - пространство всех переменных;

- $\partial_{x^{\mu}}, \partial_{u_{i}^{\alpha}}$ - частные производные, $1 \leqslant \mu \leqslant m, \alpha \in \mathrm{A}, i \in \mathbb{I}$;

- $D_{\mu}=\partial_{x^{\mu}}+\sum_{\alpha \in \mathrm{A}, i \in \mathbb{I}} u_{i+(\mu)}^{\alpha} \partial_{u_{i}^{\alpha}}-$ полные частные производные, $1 \leqslant \mu \leqslant m$;

- $d^{m} x=d x^{1} \wedge \cdots \wedge d x^{m}, d_{\mu} x=(-1)^{\mu-1} d x^{1} \wedge \ldots d \check{x}^{\mu} \cdots \wedge d x^{m}$, здесь и далее предполагается, что аргумент под значком $\sim$ пропущен.

Кроме того,

- $\Phi=\mathcal{C}^{\infty}(\mathrm{X} ; \mathbb{R})$ - алгебра всех гладких функций из $\mathrm{X}$ в $\mathbb{R}$;

- $\Phi^{\mathrm{A}}=\mathcal{C}^{\infty}(\mathrm{X} ; \mathrm{U})$ есть $\Phi$-модуль всех гладких функций из $\mathrm{X}$ в U; 
- $\Phi_{\mathbb{I}}^{\mathrm{A}}=\mathcal{C}^{\infty}(\mathrm{X} ; \mathbf{U})$ есть $\Phi$-модуль всех гладких функций из $\mathrm{X}$ в $\mathbf{U}$;

- $\mathcal{F}=\mathcal{C}_{\text {fin }}^{\infty}(\mathrm{XU} ; \mathbb{R})$ - алгебра всех гладких функций из XU в $\mathbb{R}$, зависящих от конечного числа аргументов (подробнее см., например, [3], [8]);

- $\mathcal{F}^{\mathrm{K}}=\mathcal{C}_{\text {fin }}^{\infty}\left(\mathrm{XU} ; \mathbb{R}^{\mathrm{K}}\right)$ есть $\mathcal{F}$-модуль всех гладких функций из ХU в $\mathbb{R}^{\mathrm{K}}$, зависящих от конечного числа аргументов, $\mathrm{K}$ - некоторое множество индексов;

- $\mathcal{F}_{\mathbb{I}}^{\mathrm{K}}=\mathcal{C}_{\text {fin }}^{\infty}\left(\mathrm{XU} ; \mathbb{R}_{\mathbb{I}}^{\mathrm{K}}\right)$ есть $\mathcal{F}$-модуль всех гладких функций из ХU в $\mathbb{R}_{\mathbb{I}}^{\mathrm{K}}$, зависящих от конечного числа аргументов.

\section{2. Соглашения.}

- Все построения здесь локальные, без явного упоминания об этом. Например, мы пишем $\varphi \in \Phi$, фактически считая, что $\varphi \in \Phi(\mathcal{O})=\mathcal{C}^{\infty}(\mathcal{O} ; \mathbb{R})$, где $\mathcal{O}$ некоторая область в Х.

- Все линейные операции выполняются над полем вещественных чисел $\mathbb{R}$, хотя это несущественно.

- Как правило, мы предполагаем суммирование по повторяющимся верхним и нижним индексам в естественных пределах.

- Фигурные скобки $\{\cdot\}$ означают симметризацию, а квадратные [·] - альтернирование (кососимметризацию).

- Угловые скобки $\langle\cdot, \cdot\rangle$ означают спаривание элементов дуальных модулей.

- Значок 。 означает композицию отображений.

- Мы предполагаем, что изучаемая дифференциальная алгебра $(\mathcal{A}, \partial)$ невырожденная (подробнее см. п. 2.6).

2.3. Дифференциальная алгебра. Дифференциальная алгебра (см., например, [9], [10]) есть пара $(\mathcal{A}, \partial)$, где

- $\mathcal{A}$ - ассоциативная коммутативная унитальная алгебра,

- $\partial=\left(\partial_{1}, \ldots, \partial_{m}\right)$ есть $\mathcal{A}$-линейно независимое подмножество из $\mathfrak{D}(\mathcal{A})$ такое, что $\left[\partial_{\mu}, \partial_{\nu}\right]=0,1 \leqslant \mu, \nu \leqslant m$.

Напомним, что для каждой ассоциативной коммутативной унитальной алгебры $\mathcal{A}$ определено множество $\mathfrak{D}(\mathcal{A})$ всех ее дифференцирований, т. е. множество всех эндоморфизмов $X \in \operatorname{End}_{\mathbb{R}}(\mathcal{A})$ таких, что для всех $a, b \in \mathcal{A}$ справедливо правило Лейбница: $X(a b)=(X a) b+a(X b)$. Множество $\mathfrak{D}(\mathcal{A})$ обладает естественной структурой алгебры Ли над алгеброй $\mathcal{A}$, т. е. имеет две согласованные структуры: структуру алгебры Ли и структуру $\mathcal{A}$-модуля, с условием согласования: $[a X, Y]=a[X, Y]-(Y a) X$ для всех $a \in \mathcal{A}$ и для всех $X, Y \in \mathfrak{D}(\mathcal{A})$.

Пусть

- $\mathfrak{d}=\left\{\zeta=\zeta^{\mu} \partial_{\mu} \mid \zeta^{\mu} \in \mathcal{A}\right\}$ есть подалгебра алгебры Ли $\mathfrak{D}(\mathcal{A})$ с $\mathcal{A}$-базисом $\partial=\left(\partial_{1}, \ldots, \partial_{m}\right)$

- $\mathfrak{d}^{*}=\operatorname{Hom}_{\mathcal{A}}(\mathfrak{d} ; \mathcal{A})=\left\{\omega=\omega_{\mu} \theta^{\mu} \mid \omega_{\mu} \in \mathcal{A}\right\}$ есть дуальный $\mathcal{A}$-модуль с дуальным $\mathcal{A}$-базисом $\theta=\left(\theta^{1}, \ldots, \theta^{m}\right)$, где $\left\langle\theta^{\mu}, \partial_{\nu}\right\rangle=\theta^{\mu}\left(\partial_{\nu}\right)=\delta_{\nu}^{\mu}$, для всех $1 \leqslant \mu, \nu \leqslant m$.

2.4. Дифференциальные идеалы. Пусть $(\mathcal{A}, \partial)$ - дифференциальная алгебра. Идеал $\mathcal{I}$ алгебры $\mathcal{A}$ называется дифберенциальным, или $(\mathcal{A}, \partial)$-идеалом, если он замкнут относительно дифференцирований $\partial_{1}, \ldots, \partial_{m}$, т. е. если $\partial(\mathcal{I}) \subset \mathcal{I}$. Ясно, что пара $(\mathcal{I}, \partial)$ - дифференциальная алгебра, где $\partial=\left.\partial\right|_{\mathcal{I}}$. 
Пусть $\mathcal{I}$ есть $(\mathcal{A}, \partial)$-идеал. Дифференциальная фактор-алгебра $(\overline{\mathcal{A}}, \bar{\partial})$ определена точной последовательностью $\mathcal{A}$-модулей

$$
0 \rightarrow \mathcal{I} \rightarrow \mathcal{A} \rightarrow \overline{\mathcal{A}} \rightarrow 0, \quad a \mapsto \bar{a}=a+\mathcal{I}
$$

и правилом $\bar{\partial}_{\mu} \bar{a}=\overline{\partial_{\mu} a}$ для всех $a \in \mathcal{A}, 1 \leqslant \mu \leqslant m$.

2.5. Дифференциальные модули. Пусть $(\mathcal{A}, \partial)$ - дифференциальная алгебра. $\mathcal{A}$-модуль $\mathcal{M}$ называется дифференииальным, или $(\mathcal{A}, \partial)$-модулем, если определен морфизм алгебр Ли $\mathfrak{d} \rightarrow \mathfrak{g l}(\mathcal{M})$ такой, что выполняется правило Лейбница $\partial_{\mu}(a M)=\left(\partial_{\mu} a\right) M+a\left(\partial_{\mu} M\right)$ для всех $1 \leqslant \mu \leqslant m, a \in \mathcal{A}, M \in \mathcal{M}$. Напомним, что $\mathfrak{g l}(\mathcal{M})$ - алгебра Ли, присоединенная к ассоциативной алгебре $\operatorname{End}_{\mathbb{R}}(\mathcal{M})$ всех линейных эндоморфизмов пространства $\mathcal{M}$.

Подмодуль $\mathcal{S}(\mathcal{A}, \partial)$-модуля $\mathcal{M}$ называется дифференииалъным, или $(\mathcal{A}, \partial)$-подмодулем, если он замкнут относительно дифференцирований $\partial_{1}, \ldots, \partial_{m}$, т. е. если $\partial(\mathcal{S}) \subset \mathcal{S}$. В этом случае дифференциальный фактор-модуль $\overline{\mathcal{M}}$ определен точной последовательностью $\mathcal{A}$-модулей

$$
0 \rightarrow \mathcal{S} \rightarrow \mathcal{M} \rightarrow \overline{\mathcal{M}} \rightarrow 0, \quad M \mapsto \bar{M}=M+\mathcal{S}
$$

и правилом $\bar{\partial}_{\mu} \bar{M}=\overline{\partial_{\mu} M}$ для всех $M \in \mathcal{A}, 1 \leqslant \mu \leqslant m$.

2.6. Дифференциальные многочлены. Пусть $(\mathcal{A}, \partial)$ - дифференциальная алгебра. Обозначим через $\mathcal{A}[\partial]$ множество всех дифберенииальных многочленов, т. е. $\mathcal{A}$-модуль всех многочленов от переменных $\partial_{1}, \ldots, \partial_{m}$ с коэффициентами из $\mathcal{A}$. Каждый многочлен $P=P(\partial) \in \mathcal{A}[\partial]$ может быть записан двумя способами:

$$
P(\partial)=\sum_{|i| \leqslant \operatorname{ord}(P)} a^{i} \partial_{i}=\sum_{0 \leqslant p \leqslant \operatorname{ord}(P)} a^{\mu_{1} \ldots \mu_{p}} \partial_{\mu_{1} \ldots \mu_{p}},
$$

где

- число $\operatorname{ord}(P) \in \mathbb{Z}_{+}$есть порядок многочлена $P(\partial)$,

- коэффициенты $a^{i}, a^{\mu_{1} \ldots \mu_{p}} \in \mathcal{A}, i \in \mathbb{I}, 1 \leqslant \mu_{1}, \ldots, \mu_{p} \leqslant m$,

- $a^{\pi\left(\mu_{1} \ldots \mu_{p}\right)}=a^{\mu_{1} \ldots \mu_{p}}$ для любой перестановки $\pi \in \Sigma_{p}$,

- моном $\partial_{i}=\left(\partial_{1}\right)^{i^{1}} \ldots\left(\partial_{m}\right)^{i^{m}}, i=\left(i^{1}, \ldots, i^{m}\right) \in \mathbb{I}$,

- моном $\partial_{\mu_{1} \ldots \mu_{p}}=\partial_{\mu_{1}} \ldots \partial_{\mu_{p}}, 1 \leqslant \mu_{1}, \ldots, \mu_{p} \leqslant m$.

Множество $\mathcal{A}[\partial]$ имеет структуру ассоциативной унитальной алгебры, причем билинейная операция задана правилом Лейбница

$$
\partial_{\mu} \circ a=\partial_{\mu} a+a \partial_{\mu} \quad \text { для всех } \quad 1 \leqslant \mu \leqslant m, a \in \mathcal{A} \text {. }
$$

Например,

$$
\left(a \partial_{\mu}\right) \circ\left(b \partial_{\nu}\right)=\left(a\left(\partial_{\mu} b\right)\right) \partial_{\nu}+(a b) \partial_{\mu \nu} \quad \text { для всех } \quad a, b \in \mathcal{A}, 1 \leqslant \mu, \nu \leqslant m .
$$

Каждый дифференциальный многочлен $P(\partial)=\sum_{i} a^{i} \partial_{i} \in \mathcal{A}[\partial]$ определяет линейный дифференциальный оператор на пространстве $\mathcal{A}$ правилом: $P(\partial) b=\sum_{i} a^{i}\left(\partial_{i} b\right)$, $b \in \mathcal{A}$. Заметим, что билинейная операция на алгебре $\mathcal{A}[\partial]$ совпадает с правилом композиции в алгебре $\operatorname{End}_{\mathbb{R}}(\mathcal{A})$. 
Дифференциальная алгебра $(\mathcal{A}, \partial)$ называется невырожденной, если для любого $(\mathcal{A}, \partial)$-идеала $\mathcal{I}$ и любого дифференциального многочлена $P(\partial)=\sum_{i} a^{i} \partial_{i} \in \mathcal{A}[\partial]$ вложение $P(\partial) \phi \in \mathcal{I}$ имеет место для всех $\phi \in \mathcal{A}$ тогда и только тогда, когда все коэффициенты $a^{i} \in \mathcal{I}$. В этом случае определено вложение $\mathcal{A}[\partial] \subset \operatorname{End}_{\mathbb{R}}(\mathcal{A})$.

Мы предполагаем, что изучаемая дифференциальная алгебра невырожденная.

Таким же образом для каждого $(\mathcal{A}, \partial)$-модуля $\mathcal{M}$ определено множество $\mathcal{M}[\partial]$ всех дифференциальных многочленов с коэффициентами из $\mathcal{M}$. В этом случае правило Лейбница задает на множестве $\mathcal{M}[\partial]$ структуру $\mathcal{A}[\partial]$-бимодуля.

2.7. Интегрирование по частям (формула Грина). Пусть $(\mathcal{A}, \partial)$ - дифференциальная алгебра, и $\mathcal{K}$ есть $(\mathcal{A}, \partial)$-модуль. Дуальный $\mathcal{A}$-модуль $\mathcal{K}^{*}=\operatorname{Hom}_{\mathcal{A}}(\mathcal{K} ; \mathcal{A})$ имеет структуру $(\mathcal{A}, \partial)$-модуля, задаваемую правилом Лейбница

$$
\partial_{\mu}\langle\phi, f\rangle=\left\langle\partial_{\mu} \phi, f\right\rangle+\left\langle\phi, \partial_{\mu} f\right\rangle \quad \text { для всех } 1 \leqslant \mu \leqslant m, \phi \in \mathcal{K}^{*}, f \in \mathcal{K} .
$$

Далее, пусть $\mathcal{K}, \mathcal{L}$ суть $(\mathcal{A}, \partial)$-модули, и $\mathcal{K}^{*}, \mathcal{L}^{*}-$ их дуальные. $\mathcal{A}$-модули $\mathcal{M}=$ $\operatorname{Hom}_{\mathcal{A}}(\mathcal{K} ; \mathcal{L})$ и $\mathcal{N}=\operatorname{Hom}_{\mathcal{A}}\left(\mathcal{L}^{*} ; \mathcal{K}^{*}\right)$ имеют структуры $(\mathcal{A}, \partial)$-модуля, также задаваемые правилом Лейбница. Например,

$$
\partial_{\mu} \circ M=\partial_{\mu} M+M \circ \partial_{\mu} \quad \text { для всех } \quad 1 \leqslant \mu \leqslant m, \quad M \in \mathcal{M} .
$$

Более того, определено сопряжение $\mathcal{M} \rightarrow \mathcal{N}, M \mapsto N=M^{*}$, где

$$
\left\langle M^{*} \phi, f\right\rangle=\langle\phi, M f\rangle \quad \text { для всех } \phi \in \mathcal{L}^{*}, f \in \mathcal{K} .
$$

Мы будем пользоваться матричными обозначениями: $\phi M=M^{*} \phi$. В этом случае предыдущее определение принимает вид $\langle\phi M, f\rangle=\langle\phi, M f\rangle$.

Для каждого многочлена $P(\partial)=\sum_{p} M^{\mu_{1} \ldots \mu_{p}} \partial_{\mu_{1} \ldots \mu_{p}} \in \mathcal{M}[\partial]$ определен сопряженный по Лагранжу многочлен

$$
P^{*}(\partial)=\sum_{p}(-1)^{p} \partial_{\mu_{1} \ldots \mu_{p}} \circ\left(M^{\mu_{1} \ldots \mu_{p}}\right)^{*} \in \mathcal{N}[\partial],
$$

T. e.

$$
P^{*}(\partial) \phi=\sum_{p}(-1)^{p} \partial_{\mu_{1} \ldots \mu_{p}}\left(\phi M^{\mu_{1} \ldots \mu_{p}}\right) \quad \text { для всех } \phi \in \mathcal{L}^{*} .
$$

ТЕоремА 1. Для каждого линейного дифференциального оператора

$$
P(\partial)=\sum_{p} M^{\mu_{1} \ldots \mu_{p}} \partial_{\mu_{1} \ldots \mu_{p}} \in \operatorname{Hom}_{\mathbb{R}}(\mathcal{K} ; \mathcal{L})
$$

существует билинейный дифференциальный оператор

$$
J(\partial)=\left(J^{\mu}(\partial)\right) \in \operatorname{Hom}_{\mathbb{R}}\left(\mathcal{L}^{*} \times \mathcal{K} ; \mathcal{A}^{m}\right)
$$

такой, что формула Грина

$$
\langle\phi, P(\partial) f\rangle-\left\langle P^{*}(\partial) \phi, f\right\rangle=\partial_{\mu}\left(J^{\mu}(\partial)(\phi, f)\right)
$$

справедлива для всех пар $(\phi, f) \in \mathcal{L}^{*} \times \mathcal{K}$. Например, можно положить

$$
J^{\mu}(\partial)(\phi, f)=\sum_{p, q}(-1)^{p}\left\langle\partial_{\mu_{1} \ldots \mu_{p}}\left(\phi M^{\mu \mu_{1} \ldots \mu_{p} \nu_{1} \ldots \nu_{q}}\right), \partial_{\nu_{1} \ldots \nu_{q}} f\right\rangle .
$$

ДокАЗАТЕЛЬСТво проводится прямой проверкой. 
2.8. Дифференциальный комплекс. Пусть $(\mathcal{A}, \partial)$ - дифференциальная алгебра. Определена внешняя алгебра $\Omega(\mathcal{A})=\oplus_{q \in \mathbb{Z}} \Omega^{q}(\mathcal{A})$, где $\mathcal{A}$-модули

$$
\Omega^{q}(\mathcal{A})= \begin{cases}\mathcal{A}, & q=0, \\ \wedge^{q} \mathfrak{d}^{*}, & 1 \leqslant q \leqslant m, \\ 0, & q<0 \text { или } q>m .\end{cases}
$$

В свою очередь, для каждого $(\mathcal{A}, \partial)$-модуля $\mathcal{M}$ определен $(\mathcal{A}, \partial)$-модуль $\Omega(\mathcal{M})=$ $\mathcal{M} \otimes_{\mathcal{A}} \Omega(\mathcal{A})$.

Каждая форма $\omega \in \Omega^{q}(\mathcal{M}), 1 \leqslant q \leqslant m$, имеет представление

$$
\omega=\frac{1}{q !} \omega_{\nu_{1} \ldots \nu_{q}} \otimes \theta^{\nu_{1} \ldots \nu_{q}}, \quad \omega_{\nu_{1} \ldots \nu_{q}} \in \mathcal{M}
$$

где

- $\omega_{\pi\left(\nu_{1} \ldots \nu_{q}\right)}=\operatorname{sign}(\pi) \omega_{\nu_{1} \ldots \nu_{q}}$ для всех перестановок $\pi \in \Sigma_{q}$,

- $\theta^{\nu_{1} \ldots \nu_{q}}=\theta^{\nu_{1}} \wedge \cdots \wedge \theta^{\nu_{q}}, 1 \leqslant \nu_{1}, \ldots, \nu_{q} \leqslant m$,

- $\left(\theta^{\nu_{1} \ldots \nu_{q}} \mid 1 \leqslant \nu_{1}<\cdots<\nu_{q} \leqslant m\right)$ есть базис $\mathcal{A}$-модуля $\Omega^{q}(\mathcal{A})$.

Определены следующие операции:

- $\varepsilon^{\mu}: \Omega^{q}(\mathcal{M}) \rightarrow \Omega^{q+1}(\mathcal{M}), \quad \omega \mapsto \theta^{\mu} \wedge \omega$

- $\partial_{\mu}: \Omega^{q}(\mathcal{M}) \rightarrow \Omega^{q}(\mathcal{M}), \quad \omega \mapsto \partial_{\mu} \omega$,

- $d^{q}=\left.d\right|_{\Omega^{q}(\mathcal{M})}=\varepsilon^{\mu} \circ \partial_{\mu}: \Omega^{q}(\mathcal{M}) \rightarrow \Omega^{q+1}(\mathcal{M}), \quad \omega \mapsto d \omega$, где $\omega=(1 / q !) \omega_{\nu_{1} \ldots \nu_{q}} \otimes \theta^{\nu_{1} \ldots \nu_{q}}, \partial_{\mu} \omega=(1 / q !)\left(\partial_{\mu} \omega_{\nu_{1} \ldots \nu_{q}}\right) \otimes \theta^{\nu_{1} \ldots \nu_{q}}, q \in \mathbb{Z}, 1 \leqslant \mu \leqslant m$.

Очевидно, $d \circ d=0$, так что определен дифференциальный комплекс $\left\{\Omega^{q}(\mathcal{M}) ; d^{q}\right\}$ с пространствами когомологий $H^{q}(\mathcal{M})=\operatorname{Ker} d^{q} / \operatorname{Im} d^{q-1}$.

Пусть $\mathcal{S}$ есть $(\mathcal{A}, \partial)$-подмодуль $(\mathcal{A}, \partial)$-модуля $\mathcal{M}$, и $\overline{\mathcal{M}}$ - соответствующий $(\mathcal{A}, \partial)$ фактор-модуль.

Стандартными методами гомологической алгебры (см., например, [11]) по точной последовательности $0 \rightarrow(\mathcal{S}, \partial) \rightarrow(\mathcal{M}, \partial) \rightarrow(\overline{\mathcal{M}}, \bar{\partial}) \rightarrow 0(\mathcal{A}, \partial)$-модулей строится точная последовательность

$$
0 \rightarrow\left\{\Omega^{q}(\mathcal{S}) ; d^{q}\right\} \rightarrow\left\{\Omega^{q}(\mathcal{M}) ; d^{q}\right\} \rightarrow\left\{\Omega^{q}(\overline{\mathcal{M}}) ; \bar{d}^{q}\right\} \rightarrow 0
$$

дифференциальных комплексов, которая в явном виде записывается как коммутативная диаграмма линейных пространств с точными строками, представленная на рис. 1.

Теорема 2. Последовательность линейных пространств

$$
\begin{aligned}
& 0 \longrightarrow H^{0}(\mathcal{S}) \longrightarrow H^{0}(\mathcal{M}) \longrightarrow H^{0}(\overline{\mathcal{M}}) \stackrel{\Delta}{\longrightarrow} \\
& \quad \stackrel{\Delta}{\longrightarrow} H^{1}(\mathcal{S}) \longrightarrow H^{1}(\mathcal{M}) \longrightarrow H^{1}(\overline{\mathcal{M}}) \stackrel{\Delta}{\longrightarrow} \cdots \\
& \cdots \stackrel{\Delta}{\longrightarrow} H^{m}(\mathcal{S}) \longrightarrow H^{m}(\mathcal{M}) \longrightarrow H^{m}(\overline{\mathcal{M}}) \longrightarrow 0
\end{aligned}
$$

точная, где связующие линейные отображения

$$
\Delta=\Delta^{q}: H^{q}(\overline{\mathcal{M}}) \rightarrow H^{q+1}(\mathcal{S}), \quad \bar{\omega} \mapsto \Delta \bar{\omega}, \quad 0 \leqslant q \leqslant m-1,
$$

действуют по правилу $\Delta \bar{\omega}=\overline{d \omega}$ для всех $\omega \in \Omega^{q}(\mathcal{M}), d \omega \in \Omega^{q+1}(\mathcal{S})$. 


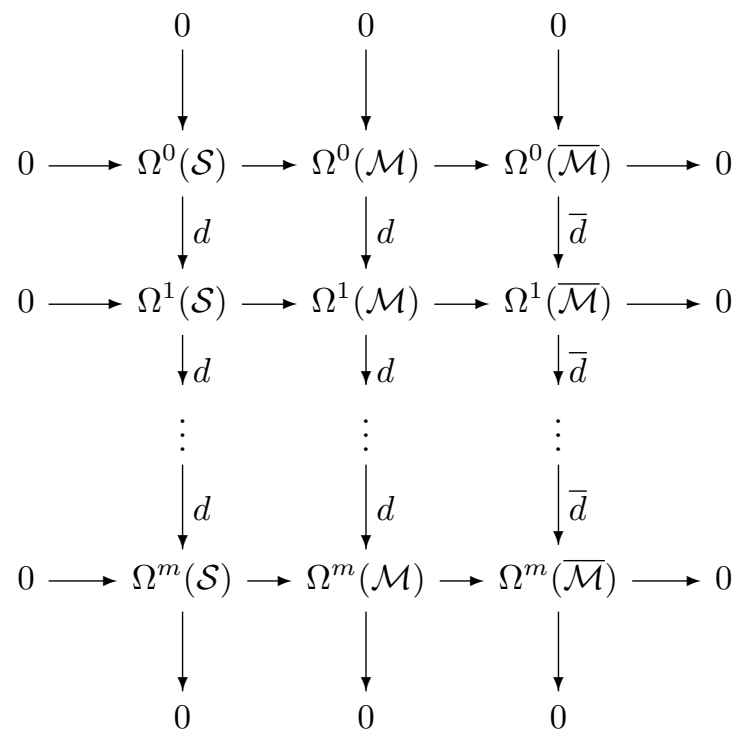

Рис. 1

ДокАЗАТЕЛЬСТво проводится простым диаграммным поиском (см., например, [11]).

Заметим, что $H^{0}(\mathcal{S})=\{\omega \in \mathcal{S} \mid d \omega=0\}, H^{0}(\mathcal{M})=\{\omega \in \mathcal{M} \mid d \omega=0\}$ и $H^{0}(\overline{\mathcal{M}})=\{\bar{\omega} \in \overline{\mathcal{M}} \mid d \omega \in \mathcal{S}\}$ суть линейные пространства дифференциальных констант $(\mathcal{A}, \partial)$-модулей $\mathcal{S}, \mathcal{M}$ и $\overline{\mathcal{M}}$.

2.9. Одна точная последовательность. Пусть $(\mathcal{A}, \partial)$ - дифференциальная алгебра, и $\mathcal{M}$ есть $(\mathcal{A}, \partial)$-модуль. Рассмотрим дифференциальный комплекс $\left.\left\{\Omega^{q}(\mathcal{M}[\partial]) ; d\right)\right\}$. В этом случае для формы

$$
\omega=\frac{1}{q !} \sum_{p} \omega_{\nu_{1} \ldots \nu_{q}}^{\mu_{1} \ldots \mu_{p}} \partial_{\mu_{1} \ldots \mu_{p}} \otimes \theta^{\nu_{1} \ldots \nu_{q}} \in \Omega^{q}(\mathcal{M}[\partial]), \quad \omega_{\nu_{1} \ldots \nu_{q}}^{\mu_{1} \ldots \mu_{p}} \in \mathcal{M},
$$

мы имеем

$$
\begin{aligned}
\partial_{\mu} \omega & =\frac{1}{q !} \sum_{p} \partial_{\mu} \circ \omega_{\nu_{1} \ldots \nu_{q}}^{\mu_{1} \ldots \mu_{p}} \partial_{\mu_{1} \ldots \mu_{p}} \otimes \theta^{\nu_{1} \ldots \nu_{q}}= \\
& =\frac{1}{q !} \sum_{p}\left[\left(\partial_{\mu} \omega_{\nu_{1} \ldots \nu_{q}}^{\mu_{1} \ldots \mu_{p}}\right) \partial_{\mu_{1} \ldots \mu_{p}}+\omega_{\nu_{1} \ldots \nu_{q}}^{\mu_{1} \ldots \mu_{p}} \partial_{\mu \mu_{1} \ldots \mu_{p}}\right] \otimes \theta^{\nu_{1} \ldots \nu_{q}} \\
d \omega & =\frac{1}{(q+1) !} \sum_{p}(d \omega)_{\nu_{0} \ldots \nu_{q}}^{\mu_{1} \ldots \mu_{p}} \partial_{\mu_{1} \ldots \mu_{p}} \otimes \theta^{\nu_{0} \ldots \nu_{q}} \\
(d \omega)_{\nu_{0} \ldots \nu_{q}}^{\mu_{1} \ldots \mu_{p}} & =(q+1)\left(\partial_{\left[\nu_{0}\right.} \omega_{\left.\nu_{1} \ldots \nu_{q}\right]}^{\mu_{1} \ldots \mu_{p}}+\delta_{\left[\nu_{0}\right.}^{\left\{\mu_{1}\right.} \omega_{\left.\nu_{1} \ldots \nu_{q}\right]}^{\left.\mu_{2} \ldots \mu_{p}\right\}}\right)
\end{aligned}
$$


Характеристикой многочлена $P(\partial)=\sum_{p} M^{\mu_{1} \ldots \mu_{p}} \partial_{\mu_{1} \ldots \mu_{p}} \in \mathcal{M}[\partial]$ называется элемент

$$
\chi(P(\partial))=\sum_{p}(-1)^{p} \partial_{\mu_{1} \ldots \mu_{p}} M^{\mu_{1} \ldots \mu_{p}} \in \mathcal{M} .
$$

TЕорема 3. Существуют линейные отображения $h^{q}: \Omega^{q}(\mathcal{M}[\partial]) \rightarrow \Omega^{q-1}(\mathcal{M}[\partial])$, $0 \leqslant q \leqslant m$, такие что для каждой формы $\omega \in \Omega^{q}(\mathcal{M}[\partial])$ справедлива гомотопическая формула

$$
\left(d^{q-1} \circ h^{q}+h^{q+1} \circ d^{q}\right) \omega= \begin{cases}\omega, & q \neq m, \\ \omega-\chi(\omega), & q=m .\end{cases}
$$

Например, можно положить

$$
h^{q} \omega= \begin{cases}0, & q=0 \\ \frac{1}{(q-1) !} \sum_{p}\left(h^{q} \omega\right)_{\nu_{1} \ldots \nu_{q-1}}^{\mu_{1} \ldots \mu_{p}} \partial_{\mu_{1} \ldots \mu_{p}} \otimes \theta^{\nu_{1} \ldots \nu_{q-1}}, & q>0\end{cases}
$$

$2 \partial e$

$$
\begin{aligned}
\left(h^{q} \omega\right)_{\nu_{1} \ldots \nu_{q-1}}^{\mu_{1} \ldots \mu_{p}} & =\sum_{s}(-1)^{s} c(p, q, s) \partial_{\lambda_{1} \ldots \lambda_{s}} \omega_{\mu \nu_{1} \ldots \nu_{q}-1}^{\mu \lambda_{1} \ldots \lambda_{s} \mu_{1} \ldots \mu_{p}}, \\
c(p, q, s) & =\frac{(p+1) \ldots(s+p+1)}{(p+m-q+1) \ldots(s+p+m-q+1)} .
\end{aligned}
$$

ДоКАЗАТЕЛЬСТвО проводится прямой проверкой.

Характеристическое отображение $\chi: \Omega^{m}(\mathcal{M}[\partial]) \rightarrow \Omega^{m}(\mathcal{M})$ действует по правилу

$$
\omega=\omega(\partial) \otimes \theta^{\wedge m} \mapsto \chi(\omega)=\chi(\omega(\partial)) \otimes \theta^{\wedge m},
$$

где $\omega(\partial)=\sum_{p} \omega^{\mu_{1} \ldots \mu_{p}} \partial_{\mu_{1} \ldots \mu_{p}} \in \mathcal{M}[\partial], \theta^{\wedge m}=\theta^{1} \wedge \cdots \wedge \theta^{m}$.

СлЕДСТВИЕ 1. Последователъность линейных пространств

$$
\begin{aligned}
& 0 \longrightarrow \Omega^{0}(\mathcal{M}[\partial]) \stackrel{d}{\longrightarrow} \Omega^{1}(\mathcal{M}[\partial]) \stackrel{d}{\longrightarrow} \quad \ldots \\
& \ldots \stackrel{d}{\longrightarrow} \Omega^{m-1}(\mathcal{M}[\partial]) \stackrel{d}{\longrightarrow} \Omega^{m}(\mathcal{M}[\partial]) \stackrel{\chi}{\longrightarrow} \Omega^{m}(\mathcal{M}) \longrightarrow 0
\end{aligned}
$$

точная, так что $H^{q}(\mathcal{M}[\partial])=0, q \neq m, H^{m}(\mathcal{M}[\partial])=\Omega^{m}(\mathcal{M}[\partial]) / \operatorname{ker} \chi$.

\section{3. УРАВНЕНИЯ В ЧАСТНЫХ ПРОИЗВОДНЫХ}

3.1. Уравнения в частных производных в алгебраическом подходе. В алгебраическом подходе (см. [1], [8], [12], [13]) система уравнений в частных производных записывается в виде $F=0$, где

- $F=\left(F^{\kappa}\right) \in \mathcal{F}^{\mathrm{K}}, F^{\kappa}=F^{\kappa}(x, \mathbf{u})=F^{\kappa}\left(x^{\mu}, u_{i}^{\alpha}|| i \mid \leqslant p\right), \kappa \in \mathrm{K}$,

- $x^{\mu}$ - независимые переменные, $1 \leqslant \mu \leqslant m$,

- $u_{i}^{\alpha}=\partial_{x^{i}} u^{\alpha}$ - дифференциальные переменные, $\alpha \in \mathrm{A}, i \in \mathbb{I}$,

- $\partial_{x^{i}}=\left(\partial_{x^{1}}\right)^{i^{1}} \ldots\left(\partial_{x^{m}}\right)^{i^{m}}$,

- $p \in \mathbb{N}$ - порядок системы 
(подробнее обозначения см. в п. 2.1). Многообразие решений системы $F=0$ есть множество

$$
\operatorname{Sol}(F)=\left\{(x, \mathbf{u}) \in \mathrm{X} \mathbf{U} \mid D_{i} F^{\kappa}(x, \mathbf{u})=0, i \in \mathbb{I}, \kappa \in \mathrm{K}\right\},
$$

где $D_{i}=\left(D_{1}\right)^{i^{1}} \ldots\left(D_{m}\right)^{i^{m}}$. Система $F=0$ называется регулярной, если множество $\operatorname{Sol}(F)$ имеет структуру гладкого подмногообразия в $X \mathbf{U}$. Функция $\phi=\left(\phi^{\alpha}\right) \in \Phi^{\mathrm{A}}$ есть решение системы $F=0$, если

$$
\left.\left.F^{\kappa}(x, \mathbf{u})\right|_{\mathbf{u}=\mathbf{j} \phi(x)} \equiv 0, \quad \text { т. e. } \quad\{(x, \mathbf{u}) \mid \mathbf{u}=\mathbf{j} \phi(x))\right\} \subset \operatorname{Sol}(F)
$$

где $ә ж е т \mathbf{j} \phi=\left(\phi_{i}^{\alpha}\right) \in \Phi_{\mathbb{I}}^{\mathrm{A}}, \phi_{i}^{\alpha}=\partial_{x^{i}} \phi^{\alpha}$.

Мы предполагаем, что система $F=0$ нетривиальная, т. е. $\operatorname{Sol}(F) \neq \mathrm{XU}$, и совместная, т. е. $\operatorname{Sol}(F) \neq \varnothing$.

3.2. Дифференциальный идеал. Пара $(\mathcal{F}, D)$ имеет структуру дифференциальной алгебры (см. п. 2.3), где $D=\left(D_{1}, \ldots, D_{m}\right)$ (заметим, что $\left[D_{\mu}, D_{\nu}\right]=0$, $1 \leqslant \mu, \nu \leqslant m)$. Более того, можно проверить, что эта алгебра невырожденная и подалгебра ее дифференциальных констант $H^{0}(\mathcal{F})=\mathbb{R}$.

Каждая система $F=0$ задает $(\mathcal{F}, D)$-идеал алгебры $(\mathcal{F}, D)$, а именно идеал

$$
\mathcal{I}=\mathcal{I}(F)=\left\{\phi=P(D) F=P_{\kappa}(D) F^{\kappa} \mid P(D)=\left(P_{\kappa}(D)\right) \in \mathcal{F}_{\mathrm{K}}[D]\right\} .
$$

По определению имеется вложение $\mathcal{I} \subset\left\{\phi \in \mathcal{F}|\phi|_{S o l(F)}=0\right\}$ (обратное вложение имеет место, если система регулярная). Идеал $\mathcal{I}$ нетривиальный, т. е. $\mathcal{I} \neq 0, \mathcal{F}$ (напомним, мы предполагаем, что система $F=0$ нетривиальная). В частности, подалгебра его дифференциальных констант $H^{0}(\mathcal{I})=0$.

Фактор-алгебра $\overline{\mathcal{F}}$ определена точной последовательностью

$$
0 \rightarrow \mathcal{I} \stackrel{\iota}{\rightarrow} \mathcal{F} \rightarrow \overline{\mathcal{F}} \rightarrow 0 .
$$

Пара $(\overline{\mathcal{F}}, \bar{D})$ имеет структуру дифференциальной алгебры, где $\bar{D}_{\mu} \bar{\phi}=\overline{D_{\mu} \phi}, 1 \leqslant \mu \leqslant$ $m, \phi \in \mathcal{F}$. Элементы алгебры $\overline{\mathcal{F}}$ можно рассматривать как гладкие функции на многообразии решений $\operatorname{Sol}(F)$ (это действительно так, если система регулярная).

3.3. Вариационный комплекс. Комплекс $\left\{\Omega^{q}(\mathcal{F}) ; d^{q}\right\}$ является одним из главных элементов формального вариационного исчисления и известен как вариационный комплекс. Здесь

$$
\mathfrak{d}=\left\{\zeta=\zeta^{\mu} D_{\mu} \mid \zeta^{\mu} \in \mathcal{F}\right\}, \quad \mathfrak{d}^{*}=\left\{\omega=\omega_{\nu} d x^{\nu} \mid \omega_{\nu} \in \mathcal{F}\right\},
$$

$\theta^{\nu}=d x^{\nu}$ (заметим, что $d x^{\nu}\left(D_{\mu}\right)=D_{\mu} x^{\nu}=\delta_{\mu}^{\nu}$ ). Следующая теорема, возможно, есть главный результат формального вариационного исчисления (см., например, [8], [12]).

ТеоремА 4. Линейнъе пространства когомологий комплекса $\left\{\Omega^{q}(\mathcal{F}) ; d^{q}\right\}$ имеют вид

$$
H^{0}(\mathcal{F})=\mathbb{R}, H^{q}(\mathcal{F})=0,1 \leqslant q \leqslant m-1, H^{m}(\mathcal{F})=\mathfrak{L}(\mathcal{F})=\mathcal{F} / \text { Ker E, }
$$

где оператор Эйлера $\mathrm{E}: \mathcal{F} \rightarrow \mathcal{F}_{\mathrm{A}}, f \mapsto \mathrm{E} f=\left(\mathrm{E}_{\alpha} f\right)$,

$$
\mathrm{E}_{\alpha} f=\delta_{u^{\alpha}} f=\sum_{i}(-1)^{|i|} D_{i} \partial_{u_{i}^{\alpha}} f, \quad \alpha \in \mathrm{A} .
$$


3.4. Законы сохранения и дифференциальные идеалы. Пусть $F=0$ есть система уравнений в частных производных, и $\mathcal{I}$ - ее дифференциальный идеал.

Для фактор-формы $\bar{\omega} \in \Omega^{m-1}(\overline{\mathcal{F}})$ условие $\bar{d} \bar{\omega}=0$ означает, что дифференциал $d \omega \in \Omega^{m}(\mathcal{I})$, и, значит, $\left.d \omega\right|_{S o l(F)}=0$, т. е. форма $\omega$ замкнута на многообразии решений. В физике такие $(m-1)$-формы называются сохраняющимися токами, а их факторы называются законами сохранения. Имея это в виду, элементы пространств когомологий $H^{q}(\overline{\mathcal{F}})$ можно рассматривать как $q$-мерные законы сохранения. При $q=m-1$ имеем обычные законы сохранения, а при $q<m-1$ имеем маломерные законы.

Таким образом, мы рассматриваем линейные пространства

$$
\mathrm{CL}^{0}(F)=H^{0}(\overline{\mathcal{F}}) / \mathbb{R}, \quad \mathrm{CL}^{q}(F)=H^{q}(\overline{\mathcal{F}}), \quad 1 \leqslant q \leqslant m-1,
$$

как пространства законов сохранения системы $F=0$.

Далее, точная последовательность $0 \rightarrow \mathcal{I} \stackrel{\iota}{\rightarrow} \mathcal{F} \rightarrow \overline{\mathcal{F}} \rightarrow 0$ порождает точную последовательность комплексов (см. рис. 1), где $\mathcal{S}=\mathcal{I}, \mathcal{M}=\mathcal{F}, \overline{\mathcal{M}}=\overline{\mathcal{F}}, H^{0}(\mathcal{I})=0$, $H^{0}(\mathcal{F})=\mathbb{R}$.

Теорема 5. Линейные пространства когомологий комплексов $\left\{\Omega^{q}(\mathcal{I}) ; d^{q}\right\}$ u $\left\{\Omega^{q}(\overline{\mathcal{F}}) ; d^{q}\right\}$ связаны следуюшими соотношениями:

$$
\begin{aligned}
& \Delta: \mathrm{CL}^{q}(F) \simeq H^{q+1}(\mathcal{I}), \quad 0 \leqslant q \leqslant m-2, \\
& 0 \rightarrow \mathrm{CL}^{m-1}(F) \stackrel{\Delta}{\rightarrow} H^{m}(\mathcal{I}) \stackrel{\iota}{\rightarrow} H^{m}(\mathcal{F}) \rightarrow H^{m}(\overline{\mathcal{F}}) \rightarrow 0, \\
& \Delta: H^{q}(\mathcal{F}) \rightarrow H^{q+1}(\mathcal{I}), \quad \bar{\omega} \mapsto \Delta \bar{\omega}=\overline{d \omega} .
\end{aligned}
$$

В частности, $\mathrm{CL}^{m-1}(F) \simeq \operatorname{ker} \overline{\mathrm{E}}$.

ДокАЗАтЕЛьство. Достаточно воспользоваться теоремами 2 и 4.

Отметим, что здесь Е: $\Omega^{m}(\mathcal{F}) \rightarrow \mathcal{F}_{\mathrm{A}}, f d^{m} x \mapsto \mathrm{E} f$ (ср. с теоремой 4), а факторотображение $\overline{\mathrm{E}}: H^{m}(\mathcal{I}) \rightarrow \mathcal{F}_{\mathrm{A}}, \overline{f d^{m} x} \mapsto \mathrm{E} f\left(\mathrm{E}\left(d \Omega^{m-1}(\mathcal{I})\right)=0\right)$.

3.5. Дифференциальные тождества. Пусть $F=0$ - система уравнений в частных производных. По определению дифференциального идеала $\mathcal{I}(F)$ имеется сюръекция $(\mathcal{F}, D)$-модулей $F: \mathcal{F}_{\mathrm{K}}[D] \rightarrow \mathcal{I}, P(D) \mapsto P(D) F$. Ядро $\mathrm{DI}=\mathrm{DI}(F)$ этой сюръекции задается точной последовательностью $(\mathcal{F}, D)$-модулей

$$
0 \rightarrow \mathrm{DI} \stackrel{\iota}{\rightarrow} \mathcal{F}_{K}[D] \stackrel{F}{\rightarrow} \mathcal{I} \rightarrow 0 .
$$

Элементы модуля DI называются дифференциальными тождествами системы $F=0$. Опять определена точная последовательность комплексов (см. рис. 1 ), где $\mathcal{S}=\mathrm{DI}$, $\mathcal{M}=\mathcal{F}_{\mathrm{K}}[D], \overline{\mathcal{M}}=\mathcal{I}$.

ТЕОРема 6. Пространства когомологий комплексов $\left\{\Omega^{q}(\mathcal{I}) ; d^{q}\right\}$ u $\left\{\Omega^{q}(\mathrm{DI}) ; d^{q}\right\}$ связаны следующими соотношениями:

$$
\begin{aligned}
H^{0}(\mathrm{DI})=0, \quad 0 & \rightarrow H^{q}(\mathcal{I}) \stackrel{\Delta}{\rightarrow} H^{q+1}(\mathrm{DI}) \rightarrow 0, \quad 0 \leqslant q \leqslant m-2, \\
& 0 \rightarrow H^{m-1}(\mathcal{I}) \stackrel{\Delta}{\rightarrow} H^{m}(\mathrm{DI}) \stackrel{\iota}{\rightarrow} H^{m}\left(\mathcal{F}_{\mathrm{K}}[D]\right) \stackrel{F}{\rightarrow} H^{m}(\mathcal{I}) \rightarrow 0 .
\end{aligned}
$$

В частности, $H^{m-1}(\mathcal{I}) \simeq \operatorname{ker} \bar{\chi}$. 
ДокАЗАтЕльство. Достаточно использовать теорему 1 и следствие 1.

Отметим, что здесь $\chi: \Omega^{m}\left(\mathcal{F}_{\mathrm{K}}[D]\right) \rightarrow \mathcal{F}_{\mathrm{K}}, P(D) d^{m} x \mapsto \chi(P(D))$ (cр. с п. 2.9), а факторотображение $\bar{\chi}: H^{m}(\mathrm{DI}) \rightarrow \mathcal{F}_{\mathrm{K}}, \overline{P(D) d^{m} x} \mapsto \chi(P(D))\left(\right.$ ясно, что $\left.\chi\left(d \Omega^{m-1}(\mathrm{DI})\right)=0\right)$.

3.6. Законы сохранения и дифференциальные тождества. Пусть $F=0$ система уравнений в частных производных, а $\mathcal{I}$ - ее дифференциальный идеал.

Теорема 7. Справедливы следующие соотношения:

$$
\mathrm{CL}^{q}(F) \simeq \begin{cases}H^{q+2}(\mathrm{DI}), & 0 \leqslant q \leqslant m-3, \\ \operatorname{ker} \bar{\chi}, & q=m-2, \\ \operatorname{ker} \overline{\mathrm{E}}, & q=m-1,\end{cases}
$$

где $\bar{\chi}: H^{m}(\mathrm{DI}) \rightarrow \mathcal{F}_{\mathrm{K}}, \bar{E}: H^{m}(\mathcal{I}) \rightarrow \mathcal{F}_{\mathrm{A}}$.

ДоКАЗАТЕЛЬСтво. Достаточно использовать теоремы 5 и 6.

Поясним, что в формулировке теоремы 7

- изоморфизмы $\mathrm{CL}^{q}(F) \simeq H^{q+2}(\mathrm{DI}), 0 \leqslant q \leqslant m-3$, действуют по правилу

$$
\mathrm{CL}^{q}(F) \ni \bar{\omega} \mapsto \overline{d \omega}=\overline{P(D) F} \mapsto \overline{d P(D)} \in H^{q+2}(\mathrm{DI}),
$$

где $\omega \in \Omega^{q}(\mathcal{F}), d \omega=P(D) F \in \Omega^{q+1}(\mathcal{I}), P(D) \in \Omega^{q+1}\left(\mathcal{F}_{\mathrm{K}}[D]\right), d P(D) F=0$, $d P(D) \in \Omega^{q+2}(\mathrm{DI})$

- изоморфизм $\mathrm{CL}^{m-2}(F) \simeq \operatorname{ker} \bar{\chi}$ действует по правилу

$$
\mathrm{CL}^{m-2}(F) \ni \bar{\omega} \mapsto \overline{d \omega}=\overline{P(D) F} \mapsto \overline{d P(D)} \in \operatorname{ker} \bar{\chi} \subset H^{m}(\mathrm{DI}),
$$

где $\omega \in \Omega^{m-2}(\mathcal{F}), d \omega=P(D) F \in \Omega^{m-1}(\mathcal{I}), P(D) \in \Omega^{m-1}\left(\mathcal{F}_{\mathrm{K}}[D]\right), d P(D) F=$ $0, \overline{d P(D)} \in \operatorname{ker} \bar{\chi}=\left\{\overline{Q(D)} \in H^{m}(\mathrm{DI}) \mid \chi(Q(D))=0\right\}$;

- изоморфизм $\mathrm{CL}^{m-1}(F) \simeq \operatorname{ker} \overline{\mathrm{E}}$ действует по правилу

$$
\mathrm{CL}^{m-1}(F) \ni \bar{\omega} \mapsto \overline{d \omega} \in \operatorname{ker} \overline{\mathrm{E}} \subset H^{m}(\mathcal{I}),
$$

где $\omega \in \Omega^{m-1}(\mathcal{F}), d \omega \in \Omega^{m}(\mathcal{I}), \operatorname{ker} \overline{\mathrm{E}}=\left\{\bar{\phi} \in H^{m}(\mathcal{I}) \mid \mathrm{E} \phi=0\right\}$.

Опишем подробнее ядро ker $\overline{\mathrm{E}}$. Прежде всего, $\Omega^{m}\left(\mathcal{F}_{\mathrm{K}}[D]\right)=\mathcal{F}_{\mathrm{K}}[D] \otimes d^{m} x$, так что можно отождествлять пространства $\Omega^{m}\left(\mathcal{F}_{\mathrm{K}}[D]\right)$ и $\mathcal{F}_{\mathrm{K}}[D]$. Далее, в силу формулы Грина (см. п. 2.7) каждая форма $Q(D) \in \Omega^{m}\left(\mathcal{F}_{\mathrm{K}}[D]\right)$ имеет представление $Q(D)=$ $\chi+d J(D)$, где

- $Q(D)=\sum_{p} Q^{\mu_{1} \ldots \mu_{p}} D_{\mu_{1} \ldots \mu_{p}}$

- $\chi=\chi(Q(D))=Q^{*}(D) 1 \in \mathcal{F}_{\mathrm{K}}, \chi F=\langle 1 Q(D), F\rangle \in \mathcal{F}$,

- $J(D)=J^{\mu}(D) d_{\mu} x \in \Omega^{m-1}\left(\mathcal{F}_{\mathrm{K}}[D]\right)$,

$$
J^{\mu}(D)=\sum_{p, q}(-1)^{p} D_{\mu_{1} \ldots \mu_{p}} \circ Q^{\mu \mu_{1} \ldots \mu_{p} \nu_{1} \ldots \nu_{q}} D_{\nu_{1} \ldots \nu_{q}} \text {. }
$$

В свою очередь,

$$
\begin{aligned}
\mathrm{E}_{\alpha}(\chi F) & =\sum_{i}(-1)^{|i|} D_{i} \partial_{u_{i}^{\alpha}}\left(\chi_{\kappa} F^{\kappa}\right)= \\
& =\sum_{i}(-1)^{|i|} D_{i}\left(\left(\partial_{u_{i}^{\alpha}} \chi_{\kappa}\right) F^{\kappa}\right)+\sum_{i}(-1)^{|i|} D_{i}\left(\left(\partial_{u_{i}^{\alpha}} F^{\kappa}\right) \chi_{\kappa}\right)= \\
& =\left(\chi^{*} F\right)_{\alpha}+\left(F^{*} \chi\right)_{\alpha}, \quad \alpha \in \mathrm{A},
\end{aligned}
$$


где $\left(\right.$ см. [13]) $\chi^{*} \in \operatorname{Hom}_{\mathbb{R}}\left(\mathcal{F}^{\mathrm{K}} ; \mathcal{F}_{\mathrm{A}}\right)$ - оператор, сопряженный по Лагранжу к оператору универсальной линеаризации

$$
\chi_{*} \in \operatorname{Hom}_{\mathbb{R}}\left(\mathcal{F}^{\mathrm{A}} ; \mathcal{F}_{\mathrm{K}}\right), \quad\left(\chi_{*} f\right)_{\kappa}=\sum_{i}\left(\partial_{u_{i}^{\alpha}} \chi_{\kappa}\right) D_{i} f^{\alpha}, \quad f \in \mathcal{F}^{\mathrm{A}}, \quad \kappa \in \mathrm{K},
$$

a $F^{*} \in \operatorname{Hom}_{\mathbb{R}}\left(\mathcal{F}_{\mathrm{K}} ; \mathcal{F}_{\mathrm{A}}\right)$ - оператор, сопряженный по Лагранжу к оператору универсальной линеаризации

$$
F_{*} \in \operatorname{Hom}_{\mathbb{R}}\left(\mathcal{F}^{\mathrm{A}} ; \mathcal{F}^{K}\right), \quad\left(F_{*} \phi\right)^{\kappa}=\sum_{i}\left(\partial_{u_{i}^{\alpha}} F^{\kappa}\right) D_{i} \phi^{\alpha}, \quad \phi \in \mathcal{F}^{\mathrm{A}}, \quad \kappa \in \mathrm{K} .
$$

Таким образом,

$$
\operatorname{ker} \bar{E} \simeq\left\{\chi \in \mathcal{F}_{\mathrm{K}} \mid \chi^{*} F+F^{*} \chi=0\right\} \text {, }
$$

в частности $\left.F^{*} \chi\right|_{S o l(F)}=0$ для всех $\chi \in \operatorname{ker} \bar{E}$.

\section{7. Примеры.}

3.7.1. Уравнения Коши-Римана. Здесь мы используем комплексные обозначения:

- $i=\left(i^{1}, \ldots, i^{m}\right), j=\left(j^{1}, \ldots, j^{m}\right) \in \mathbb{Z}_{+}^{m}=\mathbb{I}, m \geqslant 2$;

- $z=\left(z^{1}, \ldots, z^{m}\right), \bar{z}=\left(\bar{z}^{1}, \ldots, \bar{z}^{m}\right) \in \mathbb{C}^{m}=\mathrm{X}, \operatorname{dim}_{\mathbb{R}} X=2 m$;

- $w, \bar{w} \in \mathbb{C}=\mathrm{U}, \operatorname{dim}_{\mathbb{R}} \mathrm{U}=2, \quad \mathbf{w}=\left(w_{i j}\right), \quad \overline{\mathbf{w}}=\left(\bar{w}_{i j}\right) \in \mathbb{C}_{\mathbb{I} \times \mathbb{I}}=\mathbf{U}$;

- $D_{\mu}=\partial_{z^{\mu}}+\sum_{i, j}\left(w_{i+(\mu), j} \partial_{w_{i j}}+\bar{w}_{i+(\mu), j} \partial_{\bar{w}_{i j}}\right)$, $\bar{D}_{\mu}=\partial_{\bar{z}^{\mu}}+\sum_{i, j}\left(w_{i, j+(\mu)} \partial_{w_{i j}}+\bar{w}_{i, j+(\mu)} \partial_{\bar{w}_{i j}}\right)$, $D_{i} \bar{D}_{j} w=w_{i j}, \quad D_{i} \bar{D}_{j} \bar{w}=\bar{w}_{i j}, \quad \bar{w}_{i j}=\bar{w}_{j i}$;

- $\mathcal{F}=\mathcal{C}_{\text {fin }}^{\infty}(\mathrm{XU} ; \mathbb{C}), \mathcal{F} \ni \phi=\phi(z, \bar{z}, \mathbf{w}, \overline{\mathbf{w}})$.

Система уравнений Коши-Римана записывается следующим образом:

$$
C R=\left\{\partial_{\bar{z}^{\mu}} w=0,1 \leqslant \mu \leqslant m\right\}=\left\{w_{0(\mu)}=0, \bar{w}_{(\mu) 0}=0,1 \leqslant \mu \leqslant m\right\},
$$

т. е. здесь $F=\left(F_{\mu}, \bar{F}_{\mu}\right) \in \mathcal{F}_{m}, F_{\mu}=w_{0,(\mu)}, \bar{F}_{\mu}=\bar{w}_{(\mu), 0,}, 1 \leqslant \mu \leqslant m$.

Многообразие решений имеет вид

$$
\operatorname{Sol}(C R)=\left\{(z, \mathbf{w}) \in \mathrm{X} \mathbf{U} \mid w_{i, j+(\mu)}=0, \bar{w}_{i+(\mu), j}=0, i, j \in \mathbb{I}, 1 \leqslant \mu \leqslant m\right\},
$$

а дифференциальный идеал -

$$
\mathcal{I}=\mathcal{I}(C R)=\left\{f \in \mathcal{F} \mid f=P^{\mu} w_{0(\mu)}+\bar{P}^{\mu} \bar{w}_{(\mu) 0}\right\}=\{f \in \mathcal{F} \mid f=P F\},
$$

где дифференциальные многочлены $P^{\mu}, \bar{P}^{\mu} \in \mathcal{F}[D, \bar{D}], P=\left(P^{\mu}, \bar{P}^{\mu}\right) \in \mathcal{F}^{m}[D, \bar{D}]$.

Рассмотрим форму $\omega=\phi d^{m} z \in \Omega^{m}(\mathcal{F})$, где $\phi=\phi(z, \mathbf{w}) \in \mathcal{F} \backslash \mathcal{I}$ (т. е. $\partial_{\bar{z}^{\mu}} \phi=0$, $\left.\partial_{\bar{w}_{i j}} \phi=0,\left.\phi\right|_{\operatorname{Sol}(C R)} \neq 0\right)$. Здесь $d \omega=\left(\bar{D}_{\mu} \phi\right) d \bar{z}^{\mu} \wedge d^{m} z$, где

$$
\begin{aligned}
\bar{D}_{\mu} \phi & =\sum_{i, j}\left(\partial_{w_{i j}} \phi\right) w_{i, j+(\mu)}=p(D, \bar{D}) w_{0,(\mu)}=p(D, \bar{D}) F_{\mu}, \\
p(D, \bar{D}) & =\sum_{i, j}\left(\partial_{w_{i j}} \phi\right) D_{i} \bar{D}_{j} \in \mathcal{F}[D, \bar{D}],
\end{aligned}
$$


так что $d \omega=P^{\mu}(D, \bar{D}) F_{\mu} \in \Omega^{m+1}(\mathcal{I}), P^{\mu}(D, \bar{D})=p(D, \bar{D}) d \bar{z}^{\mu} \wedge d^{m} z$. Класс эквивалентности $[\omega]=\omega+\Omega^{m}(\mathcal{I})+d \Omega^{m-1}(\mathcal{F}) \in \mathrm{CL}^{m}(C R)$ нетривиальный, т. е. $[\omega] \neq 0$, если вариационная производная

$$
\delta_{w} \phi=\sum_{i, j}(-1)^{|i+j|} D_{i} \bar{D}_{j}\left(\partial_{w_{i j}} \phi\right) \neq 0
$$

(cp. с теоремой 4 , заметим, что $\left.\delta_{\bar{w}} \phi=0\right)$. Далее, $d P=\left(d P^{\mu}\right) \in \Omega^{m+2}(\mathrm{DI})$, и класс $[d P]=d P+d \Omega^{m+1}(\mathrm{DI}) \in H^{m+2}(\mathrm{DI})$ нетривиальный, если класс $[\omega]$ нетривиальный, где $d P^{\mu}=\left(\bar{D}_{\nu} \circ p(D, \bar{D})\right) d \bar{z}^{\nu} \wedge d \bar{z}^{\mu} \wedge d^{m} z$.

В частности, положим $\phi=w \in \mathcal{F}$, тогда $\omega=w d^{m} z, \delta_{w} w=1 \neq 0, d \omega=w_{0,(\mu)} d \bar{z}^{\mu} \wedge$ $d^{m} z, P^{\mu}=d \bar{z}^{\mu} \wedge d^{m} z, d P^{\mu}=\bar{D}_{\nu} d \bar{z}^{\nu} \wedge d \bar{z}^{\mu} \wedge d^{m} z$.

Итак,

$$
0 \neq \mathrm{CL}^{m}(C R) \simeq H^{m+2}(\mathrm{DI}), \quad m<2 m-1 \text { для } m \geqslant 2 .
$$

3.7.2. Уравнения Янга-Миллса. Здесь $X=\mathbb{R}^{m}, U=\mathfrak{A}_{m}, \mathbf{U}=\mathfrak{A}_{m \times \mathbb{I}}, \mathbb{I}=\mathbb{Z}_{+}^{m}$, $\mathcal{F}=\mathcal{C}_{\text {fin }}^{\infty}(X \mathbf{U} ; \mathbb{R}), \Phi=\mathcal{C}_{\text {fin }}^{\infty}(X \mathbf{U} ; \mathfrak{A})$, где $\mathfrak{A}-$ алгебра Ли со скобкой $[\cdot, \cdot]$ и метрикой Киллинга $(\cdot, \cdot)$,

$$
([A, B], C)+(B,[A, C])=0 \quad \text { для всех } \quad A, B, C \in \mathfrak{A} .
$$

Подробнее,

- $U=\left\{A=\left(A_{\mu}\right) \mid A_{\mu} \in \mathfrak{A}, 1 \leqslant \mu \leqslant m\right\}$,

- $\mathbf{U}=\left\{\mathbf{A}=\left(A_{\mu, i}\right) \mid A_{\mu, i} \in \mathfrak{A}, 1 \leqslant \mu \leqslant m, i \in \mathbb{I}\right\}$.

Далее,

- $g=\left\|g_{\mu \nu}\right\|=\operatorname{diag}(1,-1, \ldots,-1)$ - метрика Минковского на $\mathbb{R}^{m}$, $g^{-1}=\left\|g^{\mu \nu}\right\|=g$

- $D_{\lambda} \in \operatorname{End}_{\mathbb{R}}(\mathcal{F}), \operatorname{End}_{\mathbb{R}}(\Phi), 1 \leqslant \lambda \leqslant m$, суть полные частные производные, $D_{\lambda} A_{\mu, i}=A_{\mu, i+(\lambda)}$

- $\nabla_{\lambda} \in \operatorname{End}_{\mathbb{R}}(\Phi), 1 \leqslant \lambda \leqslant m$, суть ковариантные производные, $\nabla_{\lambda} f=D_{\lambda} f+\left[A_{\lambda}, f\right], f \in \Phi$

- $F=F_{\mu \nu} d x^{\mu} \wedge d x^{\nu}$ - форма кривизны, где $1 \leqslant \mu, \nu \leqslant m$, $F_{\mu \nu}=\left[\nabla_{\mu}, \nabla_{\nu}\right]=D_{\mu} A_{\nu}-D_{\nu} A_{\mu}+\left[A_{\mu}, A_{\nu}\right] \in \operatorname{End}_{\mathcal{F}}(\Phi)$

- $\mathcal{L}=\left(F_{\mu \nu}, F^{\mu \nu}\right) / 4$ - лагранжиан Янга-Миллса, $F^{\mu \nu}=g^{\mu \mu^{\prime}} g^{\nu \nu^{\prime}} F_{\mu^{\prime} \nu^{\prime}}$.

Система уравнений Янга-Миллса $Y M=0$ есть система уранений Эйлера-Лагранжа для лагранжиана $\mathcal{L}$, т. е.

$$
Y M=\left(Y M^{\nu}\right), \quad Y M^{\nu}=\delta_{A_{\nu}} \mathcal{L}=\nabla_{\mu} F^{\mu \nu} \in \Phi, \quad 1 \leqslant \nu \leqslant m .
$$

В частности,

$$
\begin{aligned}
& \operatorname{Sol}(Y M)=\left\{(x, \mathbf{A}) \in X \mathbf{U} \mid D_{i} Y M^{\nu}=0, i \in \mathbb{I}, 1 \leqslant \nu \leqslant m\right\} \\
& \mathcal{I}=\mathcal{I}(Y M)=\left\{f \in \mathcal{F} \mid f=(P(D), Y M)=\left(P_{\nu}(D), Y M^{\nu}\right)\right\}
\end{aligned}
$$

где

$$
P_{\nu}(D)=\sum_{i} f_{\nu}^{i} D_{i} \in \Phi[D], \quad\left(P_{\nu}(D), Y M^{\nu}\right)=\sum_{i}\left(f_{\nu}^{i}, D_{i} Y M^{\nu}\right)
$$


Имеется дифференциальное тождество, именно $\nabla_{\nu} Y M^{\nu}=0$. Действительно,

$$
\nabla_{\nu} Y M^{\nu}=\nabla_{\nu} \nabla_{\mu} F^{\mu \nu}=-\frac{1}{2}\left[\nabla_{\mu}, \nabla_{\nu}\right] F^{\mu \nu}=\left[F_{\mu \nu}, F^{\mu \nu}\right]=0 .
$$

Рассмотрим 0-форму $\omega=\mathcal{L} \in \Omega^{0}(\mathcal{F})=\mathcal{F}$. Здесь $d \omega=D_{\lambda} \mathcal{L} d x^{\lambda}$, где

$$
\begin{aligned}
D_{\lambda} \mathcal{L} & =\frac{1}{4} D_{\lambda}\left(F_{\mu \nu}, F^{\mu \nu}\right)=\left(\left(D_{\lambda} F_{\mu \nu}, F^{\mu \nu}\right)+\left(F_{\mu \nu}, D_{\lambda} F^{\mu \nu}\right)\right)= \\
& =\frac{1}{4}\left(\left(D_{\lambda} F_{\mu \nu}+\left[A_{\lambda}, F_{\mu \nu}\right], F^{\mu \nu}\right)+\left(F_{\mu \nu}, D_{\lambda} F^{\mu \nu}+\left[A_{\lambda}, F^{\mu \nu}\right]\right)\right)= \\
& =\frac{1}{4}\left(\left(\nabla_{\lambda} F_{\mu \nu}, F^{\mu \nu}\right)+\left(F_{\mu \nu}, \nabla_{\lambda} F^{\mu \nu}\right)\right)=\frac{1}{2}\left(F_{\mu \nu}, \nabla_{\lambda} F^{\mu \nu}\right), \quad 1 \leqslant \lambda \leqslant m .
\end{aligned}
$$

Пусть теперь $m=2$. Тогда

- $F_{\mu \nu}=e_{\mu \nu} F, e_{\mu \nu}-$ знак перестановки $(1,2) \mapsto(\mu, \nu)$, $F=F_{12}=D_{1} A_{2}-D_{2} A_{1}+\left[A_{1}, A_{2}\right], F^{\mu \nu}=-F_{\mu \nu}=F_{\nu \mu}$

- $\mathcal{L}=-(F, F) / 2, Y M^{1}=\nabla_{2} F, Y M^{2}=-\nabla_{1} F$;

- $D_{1} \mathcal{L}=-\left(F, \nabla_{1} F\right)=\left(F, Y M^{2}\right), D_{2} \mathcal{L}=-\left(F, \nabla_{2} F\right)=-\left(F, Y M^{1}\right)$;

- $d \mathcal{L}=\left(F, Y M^{2}\right) d x^{1}-\left(F, Y M^{1}\right) d x^{2} \in \Omega^{1}(\mathcal{I})$,

Подробнее,

$$
d \mathcal{L}=\left(p_{1 \mu}, Y M^{\mu}\right) d x^{1}+\left(p_{2 \mu}, Y M^{\mu}\right) d x^{2}=\left(P_{1}, Y M\right) d x^{1}+\left(P_{2}, Y M\right) d x^{2},
$$

где $P_{1}=\left\|p_{11} p_{12}\right\|, \quad P_{2}=\left\|p_{21} p_{22}\right\| \in \Phi_{2}[D], \quad p_{11}=0, \quad p_{12}=F, \quad p_{21}=-F, \quad p_{22}=$ $0, Y M=\left\|Y M^{1} Y M^{2}\right\|^{t} \in \Phi^{2}$. Другими словами, $d \mathcal{L}=(\chi, Y M)$, где форма $\chi=$ $P_{1} d x^{1}+P_{2} d x^{2} \in \Omega^{1}\left(\Phi_{2}[D]\right)$. Из равенства $(d \chi, Y M)=d d \mathcal{L}=0$ следует, что $d \chi=$ $-\left\|D_{1} \circ F D_{2} \circ F\right\| d^{2} x \in \Omega^{2}(\mathrm{DI})$.

Итак, класс эквивалентности $\overline{\mathcal{L}}=\mathcal{L}+\mathcal{I} \in \mathrm{CL}^{0}(Y M), \overline{\mathcal{L}} \neq 0$, и класс эквивалентности $\overline{d \chi}=d \chi+d \Omega^{1}(\mathrm{DI}) \in H^{2}(\mathrm{DI}), \overline{d \chi} \neq 0$, т. е.

$$
0 \neq \mathrm{CL}^{0}(Y M) \simeq H^{2}(\mathrm{DI}), \quad 0<2-1=1 .
$$

Отметим, что здесь $(d \chi, Y M)=-\left(F, \nabla_{\nu} Y M^{\nu}\right) d^{2} x$ в согласии с приведенной выше общей формулой $\nabla_{\nu} Y M^{\nu}=0$.

Пусть теперь $m \geqslant 2$, и предположим, что

$$
\text { cen } \mathfrak{A}=\{C \in \mathfrak{A} \mid[C, A]=0 \text { для всех } A \in \mathfrak{A}\} \neq 0 .
$$

Рассмотрим форму $\omega=(1 / 2)\left(C, F^{\mu \nu}\right) d_{\mu \nu} x \in \Omega^{m-2}(\mathcal{F})$, где $C \in \operatorname{cen} \mathfrak{A}$, a $(m-$ 2)-формы $d_{\mu \nu} x=-d_{\nu \mu} x, 1 \leqslant \mu, \nu \leqslant m$, определены равенством $d_{\mu \nu} x=(-1)^{\mu+\nu} d x^{1} \wedge$ $\ldots d \check{x}^{\mu} \ldots d \check{x}^{\nu} \ldots \wedge d x^{m}, \mu<\nu$. Здесь

$$
\begin{aligned}
d \omega & =\frac{1}{2} D_{\lambda}\left(C, F^{\mu \nu}\right) d_{\mu \nu} x=\frac{1}{2}\left(C, D_{\lambda} F^{\mu \nu}\right) d x^{\lambda} \wedge d_{\mu \nu} x=\left(C, D_{\mu} F^{\mu \nu}\right) d_{\nu} x= \\
& =\left(C, \nabla_{\mu} F^{\mu \nu}\right) d_{\nu} x=\left(C, Y M^{\nu}\right) d_{\nu} x=\left(P^{\nu}(D), Y M\right) d_{\nu} x \in \Omega^{m-1}(\mathcal{I}),
\end{aligned}
$$

где $P^{\nu}(D)=\left\|\delta_{\lambda}^{\nu} C \mid 1 \leqslant \lambda \leqslant m\right\| \in \Phi_{m}[D]$. Итак, класс эквивалентности $\bar{\omega}=\omega+$ $d \Omega^{m-3}(\mathcal{F})+\Omega^{m-2}(\mathcal{I}) \in \mathrm{CL}^{m-2}(Y M)$, а класс эквивалентности $\overline{d \chi}=d \chi+d \Omega^{m-1}(\mathrm{DI}) \in$ $H^{m}(\mathrm{DI})$, где $\chi=P^{\nu}(D) d_{\nu} x, d \chi=D_{\nu} \circ P^{\nu}(D) d^{m} x=\left\|C D_{\lambda} \mid 1 \leqslant \lambda \leqslant m\right\| d^{m} x$. $\mathrm{K}$ сожалению, нельзя утверждать, что классы $\bar{\omega}$ и $\overline{d \chi}$ нетривиальные.

Заметим, что $\left(C D_{\lambda}, Y M^{\lambda}\right)=\left(C, D_{\lambda} Y M^{\lambda}\right)=\left(C, \nabla_{\lambda} Y M^{\lambda}\right)=0$. 


\section{4. ЭВОЛЮЦИЯ СО СВЯЗЯМИ}

4.1. Эволюционные системы. Мы сохраняем введенные выше обозначения (см. п. 2.1) и вводим несколько новых. Именно,

- $\mathrm{T}=\mathbb{R}$ - пространство дополнительной независимой переменной $t$ (время);

- $\mathbf{V}=\mathbb{R}_{\mathbb{Z}_{+} \times \mathbb{I}}^{\mathrm{A}}=\left\{\mathbf{v}=\left(v_{p, i}^{\alpha}\right) \mid v_{p, i}^{\alpha} \in \mathbb{R}, \alpha \in \mathrm{A}, p \in \mathbb{Z}_{+}, i \in \mathbb{I}\right\}$ - расширенное пространство дифференциальных переменных, проекция $\Pi: \mathbf{V} \rightarrow \mathbf{U}$ задана правилом $\mathbf{v}=\left(v_{p, i}^{\alpha}\right) \mapsto \mathbf{u}=\left(u_{i}^{\alpha}\right), u_{i}^{\alpha}=v_{0, i}^{\alpha} ;$

- $\Psi=\mathcal{C}^{\infty}(\mathrm{TX} ; \mathbb{R}), \mathcal{E}=\mathcal{C}_{\text {fin }}^{\infty}(\mathrm{TXU} ; \mathbb{R}), \mathfrak{F}=\mathcal{C}_{\text {fin }}^{\infty}(\mathrm{TXV} ; \mathbb{R})$;

- $\mathrm{D}_{t}=\partial_{t}+\sum_{\alpha, p, i} v_{p+1, i}^{\alpha} \partial_{v_{p, i}^{\alpha}}, \quad \mathrm{D}_{\mu}=\partial_{x^{\mu}}+\sum_{\alpha, p, i} v_{p, i+(\mu)}^{\alpha} \partial_{v_{p, i}^{\alpha}}, 1 \leqslant \mu \leqslant m$, $\left[\mathrm{D}_{\mu}, \mathrm{D}_{\nu}\right]=0,\left[\mathrm{D}_{t}, \mathrm{D}_{\mu}\right]=0$ для всех $1 \leqslant \mu, \nu \leqslant m$.

Эволюционная система записывается как $E=0$, где $E=\left(E^{\alpha}\right)$,

$$
E^{\alpha}=u_{t}^{\alpha}-\mathrm{f}^{\alpha}=v_{1,0}^{\alpha}-\mathrm{f}^{\alpha}(t, x, \mathbf{u}), \quad \alpha \in \mathrm{A}, \quad \mathrm{f}=\left(\mathrm{f}^{\alpha}\right) \in \mathcal{E}^{\mathrm{A}} .
$$

Многообразие решений этой системы есть

$$
\operatorname{Sol}(E)=\left\{(t, x, \mathbf{v}) \in \mathrm{TXV} \mid v_{p+1, i}^{\alpha}=\mathrm{f}_{p, i}^{\alpha}, \alpha \in \mathrm{A}, p \in \mathbb{Z}_{+}, i \in \mathbb{I}\right\} \cong \mathrm{TXU},
$$

где $\mathrm{f}_{p, i}^{\alpha}=\mathrm{D}_{t}^{p} \mathrm{D}_{i} \mathrm{f}^{\alpha}$, заметим, что $\mathcal{E} \subset \mathfrak{F}$. Следовательно, система $E=0$ регулярная, и мы будем рассматривать пространство ТXU как глобальную карту на многообразии $\operatorname{Sol}(E)$. Функция $\psi \in \Psi$ есть решение системы $E=0$, если

$$
\partial_{t} \psi^{\alpha}(t, x)=\left.\mathrm{f}^{\alpha}(t, x, \mathbf{u})\right|_{\mathbf{u}=\mathbf{j} \psi(t, x)}, \quad \mathbf{j} \psi=\left(\psi_{i}^{\alpha}=\partial_{x^{i}} \psi^{\alpha}\right) \in \Psi_{\mathbb{I}}^{\mathrm{A}} .
$$

В силу регулярности системы $E=0$, дифференциальный идеал

$$
\mathcal{I}(E)=\left\{f \in \mathfrak{F}|f|_{S o l(E)}=0\right\}
$$

Более того, фактор-алгебра $\overline{\mathfrak{F}} \cong \mathcal{E}$, а дифференциальная алгебра $(\overline{\mathfrak{F}}, \overline{\mathrm{D}}) \cong(\mathcal{E}, D)$, где $\overline{\mathrm{D}}=\left(\overline{\mathrm{D}}_{t}, \overline{\mathrm{D}}_{1}, \ldots, \overline{\mathrm{D}}_{m}\right), D=\left(D_{t}, D_{1}, \ldots, D_{m}\right)$,

$$
\begin{aligned}
& D_{\mu}=\left.\mathrm{D}_{\mu}\right|_{\mathcal{E}}=\partial_{x^{\mu}}+\sum_{\alpha, i} u_{i+(\mu)}^{\alpha} \partial_{u_{i}^{\alpha}}, \quad 1 \leqslant \mu \leqslant m, \\
& D_{t}=\left.\mathrm{D}_{t}\right|_{\mathcal{E}}=\partial_{t}+\sum_{\alpha, i} \mathrm{f}_{i}^{\alpha} \partial_{u_{i}^{\alpha}}, \quad \mathrm{f}_{i}^{\alpha}=D_{i} \mathrm{f}^{\alpha} .
\end{aligned}
$$

Следовательно, $\left\{\Omega^{q}(\overline{\mathfrak{F}}) ; \bar{d}^{q}\right\} \cong\left\{\Omega^{q}(\mathcal{E}) ; d^{q}\right\}$ и $H^{q}(\overline{\mathfrak{F}})=H^{q}(\mathcal{E}), q \in \mathbb{Z}$, где

$$
\bar{d}=d t \wedge \overline{\mathrm{D}}_{t}+d x^{\mu} \wedge \overline{\mathrm{D}}_{\mu}, \quad d=d t \wedge D_{t}+d x^{\mu} \wedge D_{\mu}
$$

Введем вспомогательный комплекс $\left\{\Omega_{x}^{q}(\mathcal{E}) ; d_{x}^{q}\right\}$, где

$$
\Omega_{x}^{q}=\left\{\omega=\frac{1}{q !} \omega_{\mu_{1}, \ldots, \mu_{q}} d x^{\mu_{1}} \wedge \cdots \wedge d x^{\mu_{q}} \mid \omega_{\mu_{1} \ldots \mu_{q}} \in \mathcal{E}\right\}, \quad d_{x}=d x^{\mu} \wedge D_{\mu}
$$

(здесь переменная $t \in \mathrm{T}$ является параметром). Тогда получим представление

$$
\Omega^{q}(\mathcal{E})=\Omega_{x}^{q}(\mathcal{E}) \oplus d t \wedge \Omega_{x}^{q-1}(\mathcal{E}), \quad \omega=\omega^{\prime}+d t \wedge \omega^{\prime \prime},
$$




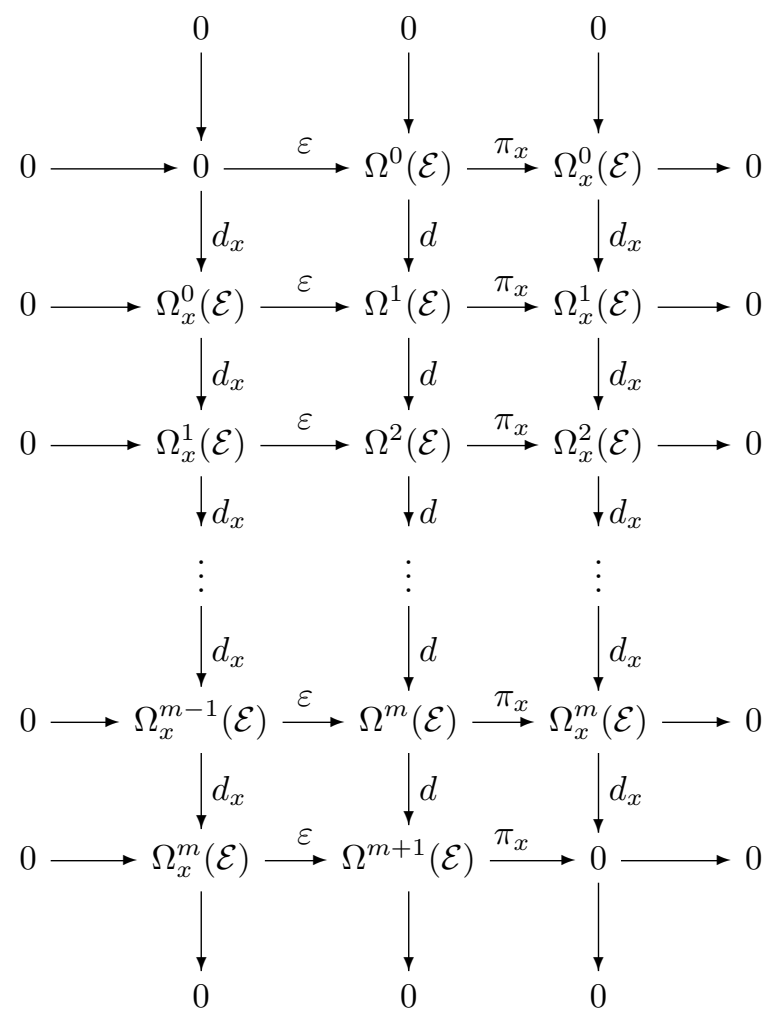

Рис. 2

где $\omega^{\prime \prime}=\pi_{t} \omega=\iota_{D_{t}} \omega \in \Omega_{x}^{q-1}(\mathcal{E}), \omega^{\prime}=\pi_{x} \omega=\omega-d t \wedge \omega^{\prime \prime} \in \Omega_{x}^{q}(\mathcal{E}), q \in \mathbb{Z}$, а дифференциал $d=d_{t}+d_{x}$,

$$
d_{t}=d t \wedge D_{t}: \Omega_{x}^{q}(\mathcal{E}) \rightarrow d t \wedge \Omega_{x}^{q}(\mathcal{E}), \quad d_{x}=d x^{\mu} \wedge D_{\mu}: \Omega_{x}^{q}(\mathcal{E}) \rightarrow \Omega_{x}^{q+1}(\mathcal{E}) .
$$

Это представление порождает точную последовательность комплексов

$$
0 \rightarrow\left\{\Omega_{x}^{q-1}(\mathcal{E}) ; d_{x}^{q-1}\right\} \stackrel{\varepsilon}{\rightarrow}\left\{\Omega^{q}(\mathcal{E}) ; d^{q}\right\} \stackrel{\pi_{x}}{\rightarrow}\left\{\Omega_{x}^{q}(\mathcal{E}) ; d_{x}^{q}\right\} \rightarrow 0,
$$

которая записывается как коммутативная диаграмма линейных пространств с точными строками, изображенная на рис. 2 , где $\varepsilon=\varepsilon^{q}: \Omega_{x}^{q}(\mathcal{E}) \rightarrow \Omega^{q+1}(\mathcal{E}), \omega^{\prime \prime} \mapsto \omega=$ $(-1)^{q} d t \wedge \omega^{\prime \prime}$. В частности, определена следующая точная последовательность линейных пространств когомологий:

$$
\begin{array}{cccc}
0 & \longrightarrow & H^{0}(\mathcal{E}) \stackrel{\bar{\pi}_{x}}{\longrightarrow} H_{x}^{0}(\mathcal{E}) \stackrel{\Delta}{\longrightarrow} \\
\stackrel{\Delta}{\longrightarrow} \quad H_{x}^{0}(\mathcal{E}) \stackrel{\bar{\varepsilon}}{\longrightarrow} H^{1}(\mathcal{E}) \stackrel{\bar{\pi}_{x}}{\longrightarrow} H_{x}^{1}(\mathcal{E}) \stackrel{\Delta}{\longrightarrow} \\
\stackrel{\Delta}{\longrightarrow} H_{x}^{1}(\mathcal{E}) \stackrel{\bar{\varepsilon}}{\longrightarrow} H^{2}(\mathcal{E}) \stackrel{\bar{\pi}_{x}}{\longrightarrow} H_{x}^{2}(\mathcal{E}) \stackrel{\Delta}{\longrightarrow} \ldots \\
\cdots \stackrel{\Delta}{\longrightarrow} H_{x}^{m-1}(\mathcal{E}) \stackrel{\bar{\varepsilon}}{\longrightarrow} H^{m}(\mathcal{E}) \stackrel{\bar{\pi}_{x}}{\longrightarrow} H_{x}^{m}(\mathcal{E}) \stackrel{\Delta}{\longrightarrow} \\
\stackrel{\Delta}{\longrightarrow} H_{x}^{m}(\mathcal{E}) \stackrel{\bar{\varepsilon}}{\longrightarrow} H^{m+1}(\mathcal{E}) \stackrel{\bar{\pi}_{x}}{\longrightarrow} 0,
\end{array}
$$


где $\Delta=\Delta^{q}: H_{x}^{q}(\mathcal{E}) \rightarrow H_{x}^{q}(\mathcal{E}), \overline{\omega^{\prime}} \mapsto \Delta \overline{\omega^{\prime}}=\overline{D_{t} \omega^{\prime}}$.

ТЕОРема 8. Для всякой эволюиионной системы $E=0$ пространства законов сохранения

$$
\mathrm{CL}^{q}(E)=0 \quad \text { для всех } 0 \leqslant q \leqslant m-1 .
$$

ДокАЗАтЕЛьство. Достаточно воспользоваться равенствами $H^{q}(\overline{\mathfrak{F}})=H^{q}(\mathcal{E})$, приведенной выше точной последовательностью и теоремой 4.

Таким образом, эволюционная система $E=0$ может иметь лишь стандартные законы сохранения $\mathrm{CL}^{m}(E)=H^{m}(\mathcal{E})=\operatorname{Ker} d^{m} / \operatorname{Im} d^{m-1}$, где

$$
\begin{aligned}
\operatorname{Ker} d^{m} & =\left\{\omega=\rho d^{m} x+d t \wedge J^{\mu} d_{\mu} x \mid \rho, J^{\mu} \in \mathcal{E}, D_{t} \rho-D_{\mu} J^{\mu}=0\right\}, \\
\operatorname{Im} d^{m-1} & =\left\{\omega=d \eta \mid \eta=j^{\mu} d_{\mu} x+d t \wedge a^{\mu \nu} d_{\mu \nu} x, j^{\mu}, a^{\mu \nu} \in \mathcal{E}\right\},
\end{aligned}
$$

так что

$$
\frac{\operatorname{Ker} d^{m}}{\operatorname{Im} d^{m-1}}=\frac{\left\{(\rho, J) \in \mathcal{E} \times \mathcal{E}^{m} \mid D_{t} \rho-D_{\mu} J^{\mu}=0\right\}}{\left\{(\rho, J) \in \mathcal{E} \times \mathcal{E}^{m} \mid \rho=D_{\mu} j^{\mu}, J^{\mu}=D_{t} j^{\mu}+D_{\nu} a^{\mu \nu}, j^{\mu}, a^{\mu \nu} \in \mathcal{E}\right\}} .
$$

ТЕОРема 9. Линейное пространство законов сохранения эволюиионной системи $E=0$ имеет вид

$$
\begin{aligned}
\mathrm{CL}^{m}(E) & =\frac{\left\{\rho \in \mathcal{E} \mid \delta_{u^{\alpha}}\left(D_{t} \rho\right)=0, \alpha \in \mathrm{A}\right\}}{\left\{\rho \in \mathcal{E} \mid \delta_{u^{\alpha}} \rho=0, \alpha \in \mathrm{A}\right\}}= \\
& =\left\{\chi \in \mathcal{E}_{\mathrm{A}} \mid \chi_{*}=\chi^{*}, D_{t} \chi+\mathrm{f}^{*} \chi=0\right\},
\end{aligned}
$$

где $\chi=\left(\chi_{\alpha}\right), \chi_{\alpha}=\delta_{u^{\alpha}} \rho, \alpha \in \mathrm{A}$,

$$
\begin{array}{ll}
\mathrm{f}^{*}: & \mathcal{E}_{\mathrm{A}} \rightarrow \mathcal{E}_{\mathrm{A}}, \quad\left(\mathrm{f}^{*} \chi\right)_{\alpha}=\sum_{\beta, i}(-1)^{|i|} D_{i}\left(\chi_{\beta} \partial_{u_{i}^{\alpha}} \mathrm{f}^{\beta}\right), \\
\chi_{*}: & \mathcal{E}^{\mathrm{A}} \rightarrow \mathcal{E}_{\mathrm{A}}, \quad\left(\chi_{*} g\right)_{\alpha}=\sum_{\beta, i}\left(\partial_{u_{i}^{\beta}} \chi_{\alpha}\right) D_{i} g^{\beta}, \quad g=\left(g^{\beta}\right) \in \mathcal{E}^{\mathrm{A}}, \\
\chi^{*}: & \mathcal{E}^{\mathrm{A}} \rightarrow \mathcal{E}_{\mathrm{A}}, \quad\left(\chi^{*} g\right)_{\alpha}=\sum_{\beta, i}(-1)^{|i|} D_{i}\left(g^{\beta} \partial_{u_{i}^{\alpha}} \chi_{\beta}\right), \quad g=\left(g^{\beta}\right) \in \mathcal{E}^{\mathrm{A}} .
\end{array}
$$

ДокАЗАТЕЛЬСтво смотри, например, в [1].

4.2. Эволюция со связями. Мы сохраняем все введенные выше обозначения.

Эволюционная система со связями $E F=0$ состоит из двух подсистем: эволюционной системы $E=0$ и системы связей $F=0$, где

$$
E=\left(E^{\alpha}=u_{t}^{\alpha}-\mathrm{f}^{\alpha}\right), \quad \mathrm{f}=\left(\mathrm{f}^{\alpha}\right) \in \mathcal{E}^{\mathrm{A}}, \quad F=\left(F^{\kappa}\right) \in \mathcal{E}^{\mathrm{K}} .
$$

Мы предполагаем, что эти подсистемы совместные. Именно, пусть

$$
\mathcal{J}=\mathcal{J}(F)=\left\{\phi=P(D) F \in \mathcal{E} \mid P(D) \in \mathcal{E}_{\mathrm{K}}[D]\right\}
$$

- дифференциальный идеал дифференциальной алгебры $(\mathcal{E}, D), D=\left(D_{1}, \ldots, D_{m}\right)$, порожденный системой связей $F=0$ (заметим, что $(\mathcal{F}, D) \subset(\mathcal{E}, D)$ ). Условие совместности в этом случае принимает вид

$$
\left.D_{t}\right|_{\mathcal{J}}: \mathcal{J} \rightarrow \mathcal{J}, \quad \text { т. е. } \quad D_{t} F=Q(D) F \in \mathcal{J}^{\mathrm{K}}
$$


Далее,

$$
\begin{aligned}
\operatorname{Sol}(E F) & =\operatorname{Sol}(E) \cap \operatorname{Sol}(F) \cong \\
& \cong\left\{(t, x, \mathbf{u}) \in \mathrm{TX} \mathbf{U} \mid D_{i} F^{\kappa}(t, x, \mathbf{u})=0, i \in \mathbb{I}, \kappa \in \mathrm{K}\right\},
\end{aligned}
$$

а пространства законов сохранения (см. п. 3.4)

$$
\mathrm{CL}^{0}(E F)=H^{0}(\overline{\mathcal{E}}) / \mathbb{R}, \quad \mathrm{CL}^{q}(E F)=H^{q}(\overline{\mathcal{E}}), \quad 1 \leqslant q \leqslant m,
$$

где $\overline{\mathcal{E}}=\mathcal{E} / \mathcal{J}$.

Как и выше, точная последовательность линейных пространств $0 \rightarrow \mathcal{J} \rightarrow \mathcal{E} \rightarrow$ $\overline{\mathcal{E}} \rightarrow 0$ порождает цепную последовательность дифференциальных комплексов (ср. с рис. 1)

$$
0 \rightarrow\left\{\Omega^{q}(\mathcal{J}) ; d^{q}\right\} \rightarrow\left\{\Omega^{q}(\mathcal{E}) ; d^{q}\right\} \rightarrow\left\{\Omega^{q}(\overline{\mathcal{E}}) ; \bar{d}^{q}\right\} \rightarrow 0
$$

и, следовательно, точную последовательность линейных пространств когомологий

$$
\begin{aligned}
0 & \longrightarrow H^{0}(\mathcal{J}) \longrightarrow H^{0}(\mathcal{E}) \longrightarrow H^{0}(\overline{\mathcal{E}}) \stackrel{\Delta}{\longrightarrow} \\
& \stackrel{\Delta}{\longrightarrow} H^{1}(\mathcal{J}) \longrightarrow H^{1}(\mathcal{E}) \longrightarrow H^{1}(\overline{\mathcal{E}}) \stackrel{\Delta}{\longrightarrow} \ldots \\
\ldots & \stackrel{\Delta}{\longrightarrow} H^{m}(\mathcal{J}) \longrightarrow H^{m}(\mathcal{E}) \longrightarrow H^{m}(\overline{\mathcal{E}}) \stackrel{\Delta}{\longrightarrow} \\
& \stackrel{\Delta}{\longrightarrow} H^{m+1}(\mathcal{J}) \longrightarrow H^{m+1}(\mathcal{E}) \longrightarrow H^{m+1}(\overline{\mathcal{E}}) \longrightarrow 0
\end{aligned}
$$

где связующие линейные отображения

$$
\Delta=\Delta^{q}: H^{q}(\overline{\mathcal{E}}) \rightarrow H^{q+1}(\mathcal{J}), \quad \bar{\omega} \mapsto \Delta \bar{\omega}, \quad 0 \leqslant q \leqslant m-1,
$$

действуют по правилу $\Delta \bar{\omega}=\overline{d \omega}$ для всех $\omega \in \Omega^{q}(\mathcal{E}), d \omega \in \Omega^{q+1}(\mathcal{J})$.

Теорема 10. Для эволюиионной системы со связями $E F=0$ справедливы следуюшие изоморфизмы:

- $\Delta: \mathrm{CL}^{q}(E F) \simeq H^{q+1}(\mathcal{J}), \quad 0 \leqslant q \leqslant m-2$,

- $\Delta: \mathrm{CL}^{m-1}(E F) \simeq H^{m}(\mathcal{J}) \cap d \Omega^{m-1}(\mathcal{E})$.

ДоКАЗАТЕЛЬСТво. Достаточно воспользоваться приведенной выше точной последовательностью и теоремой 8 . Заметим, что $H^{0}(\mathcal{J})=0$.

Теорема 11. Для эволюиионной системы со связями $E F=0$ имеет место представление

$$
\mathrm{CL}^{m}(E F) \simeq \frac{\left\{\chi \in \mathcal{E}_{\mathrm{A}} \mid \chi_{*}=\chi^{*}, D_{t} \chi+\mathrm{f}^{*} \chi=\delta_{u}(\phi F), \phi \in \mathcal{E}_{\mathrm{K}}\right\}}{\left\{\chi=\delta_{u}(\psi F) \mid \psi \in \mathcal{E}_{\mathrm{K}}\right\}} .
$$

ДОКАЗАТЕЛЬСТВО проводится путем соответствующей модификации доказательства теоремы 9. Заметим, что $\delta_{u}(\phi F)=\phi^{*} F+F^{*} \phi$ и $\delta_{u}(\psi F)=\psi^{*} F+F^{*} \psi$.

Имеется другое описание системы $E F=0$. Именно, представление

$$
\Omega^{q}(\overline{\mathcal{E}})=\Omega_{x}^{q}(\overline{\mathcal{E}}) \oplus d t \wedge \Omega_{x}^{q-1}(\overline{\mathcal{E}}), \quad \bar{\omega}=\bar{\omega}^{\prime}+d t \wedge \bar{\omega}^{\prime \prime}, \quad \bar{d}=\bar{d}_{t}+\bar{d}_{x},
$$

порождает точную последовательность комплексов (ср. с рис. 2)

$$
0 \rightarrow\left\{\Omega_{x}^{q-1}(\overline{\mathcal{E}}) ; \bar{d}_{x}^{q-1}\right\} \stackrel{\bar{\varepsilon}}{\rightarrow}\left\{\Omega^{q}(\overline{\mathcal{E}}) ; \bar{d}^{q}\right\} \stackrel{\bar{\pi}_{x}}{\longrightarrow}\left\{\Omega_{x}^{q}(\overline{\mathcal{E}}) ; \bar{d}_{x}^{q}\right\} \rightarrow 0
$$

и приводит к следующей теореме. 
ТЕорема 12. Определена точная последовательность линейных пространств

$$
\begin{aligned}
& 0 \longrightarrow H^{0}(\overline{\mathcal{E}}) \stackrel{\bar{\pi}_{x}}{\longrightarrow} H_{x}^{0}(\overline{\mathcal{E}}) \stackrel{\Delta}{\longrightarrow} \\
& \stackrel{\Delta}{\longrightarrow} H_{x}^{0}(\overline{\mathcal{E}}) \stackrel{\bar{\varepsilon}}{\longrightarrow} H^{1}(\overline{\mathcal{E}}) \stackrel{\bar{\pi}_{x}}{\longrightarrow} H_{x}^{1}(\overline{\mathcal{E}}) \stackrel{\Delta}{\longrightarrow} \\
& \stackrel{\Delta}{\longrightarrow} H_{x}^{1}(\overline{\mathcal{E}}) \stackrel{\bar{\varepsilon}}{\longrightarrow} H^{2}(\overline{\mathcal{E}}) \stackrel{\bar{\pi}_{x}}{\longrightarrow} H_{x}^{2}(\overline{\mathcal{E}}) \stackrel{\Delta}{\longrightarrow} \cdots \\
& \ldots \stackrel{\Delta}{\longrightarrow} H_{x}^{m-1}(\overline{\mathcal{E}}) \stackrel{\bar{\varepsilon}}{\longrightarrow} H^{m}(\overline{\mathcal{E}}) \stackrel{\bar{\pi}_{x}}{\longrightarrow} H_{x}^{m}(\overline{\mathcal{E}}) \stackrel{\Delta}{\longrightarrow} \\
& \stackrel{\Delta}{\longrightarrow} H_{x}^{m}(\overline{\mathcal{E}}) \stackrel{\bar{\varepsilon}}{\longrightarrow} H^{m+1}(\overline{\mathcal{E}}) \stackrel{\bar{\pi}_{x}}{\longrightarrow} 0,
\end{aligned}
$$

где $\Delta=\Delta^{q}: H_{x}^{q}(\overline{\mathcal{E}}) \rightarrow H_{x}^{q}(\overline{\mathcal{E}}), \overline{\omega^{\prime}} \mapsto \Delta \overline{\omega^{\prime}}=\overline{\omega^{\prime \prime}}=\overline{D_{t} \omega^{\prime}}$.

Для вычисления пространств $H_{x}^{q}(\overline{\mathcal{E}})$ можно воспользоваться теоремами 5,6 и 7.

\section{3. Примеры.}

4.3.1. Параболическая связь. Рассмотрим систему $E F=0$, где

$$
E=u_{t}-u_{x y}, \quad F=u_{y}-u_{x x},
$$

и форму $\omega=u d x+u_{x} d y+u_{y} d t \in \Omega^{1}(\mathcal{E})$. Дифференциал

$$
\begin{aligned}
d \omega & =\left(u_{y} d y+u_{t} d t\right) \wedge d x+\left(u_{x x} d x+u_{x t} d t\right) \wedge d y+\left(u_{x y} d x+u_{u u} d y\right) \wedge d t= \\
& =\left(-u_{y}+u_{x x}\right) d x \wedge d y+\left(u_{t}-u_{x y}\right) d t \wedge d x+\left(u_{x t}-u_{y y}\right) d t \wedge d y= \\
& =-F d x \wedge d y+d t \wedge\left(E d x+\left(\mathrm{D}_{x} E-\mathrm{D}_{y} F\right) d y\right) \in \Omega^{2}(\mathcal{J}),
\end{aligned}
$$

так что класс эквивалентности $\bar{\omega} \in \mathrm{CL}^{1}(E F)$, и, как легко проверить, $\bar{\omega} \neq 0$. Далее,

$$
\begin{aligned}
0=d d \omega & =\left(-\mathrm{D}_{t} F+\mathrm{D}_{y} E-\mathrm{D}_{x}\left(\mathrm{D}_{x} E-\mathrm{D}_{y}\right) F\right) d t \wedge d x \wedge d y= \\
& =\left(\left(\mathrm{D}_{y}-\mathrm{D}_{x} \mathrm{D}_{x}\right) E-\left(\mathrm{D}_{t}-\mathrm{D}_{x} \mathrm{D}_{x}\right) F\right) d t \wedge d x \wedge d y,
\end{aligned}
$$

т. е. класс эквивалентности $\overline{P(\mathrm{D})}=\overline{\left\|P_{F}, P_{E}\right\|} \in H^{3}(\mathrm{DI})$, где

$$
P_{F}=\left(\mathrm{D}_{y}-\mathrm{D}_{x} \mathrm{D}_{x}\right) \otimes d t \wedge d x \wedge d y, \quad P_{E}=-\left(\mathrm{D}_{t}-\mathrm{D}_{x} \mathrm{D}_{y}\right) \otimes d t \wedge d x \wedge d y .
$$

4.3.2. Эллиптическая связь. Рассмотрим систему $E F=0$,

$$
E=u_{t}-\mathrm{f}(\xi, \eta), \quad F=u_{x x}+u_{y y}, \quad \xi=u_{x}, \eta=u_{y},
$$

где $\mathrm{f}(\xi, \eta)$ - гармоническая функция (т. е. $\left.\mathrm{f}_{\xi \xi}+\mathrm{f}_{\eta \eta}=0\right)$. Пусть $A, B, C(\xi, \eta)$ - гладкие функции, удовлетворяющие уравнениям

$$
\left\{\begin{array} { l } 
{ A _ { \xi } = B _ { \eta } , } \\
{ A _ { \eta } = - B _ { \xi } , }
\end{array} \quad \left\{\begin{array}{l}
C_{\xi}=A_{\xi} \mathrm{f}_{\xi}-A_{\eta} \mathrm{f}_{\eta}, \\
C_{\eta}=B_{\eta} \mathrm{f}_{\eta}-B_{\xi} \mathrm{f}_{\xi} .
\end{array}\right.\right.
$$

Заметим, что в силу первой подсистемы функция $W(\zeta)=A(\xi, \eta)+i B(\xi, \eta)$ голоморфная, $\zeta=\xi+i \eta$, а предположение $\mathrm{f}_{\xi \xi}+\mathrm{f}_{\eta \eta}=0$ есть условие совместности для второй подсистемы. Далее, рассмотрим форму $\omega=A d x+B d y+C d t \in \Omega^{1}(\mathcal{E})$. Здесь дифференциал

$$
\begin{aligned}
d \omega= & \left(\mathrm{D}_{x} B-\mathrm{D}_{y} A\right) d x \wedge d y+d t \wedge\left(\left(\mathrm{D}_{t} A-\mathrm{D}_{x} C\right) d x+\left(\mathrm{D}_{t} B-\mathrm{D}_{y} C\right) d y\right)= \\
= & B_{\xi} F d x \wedge d y+d t \wedge\left(\left(A_{\xi} \mathrm{D}_{x} E+A_{\eta} \mathrm{D}_{y} E+A_{\eta} \mathrm{f}_{\eta} F\right) d x+\right. \\
& \left.+\left(B_{\xi} \mathrm{D}_{x} E+B_{\eta} \mathrm{D}_{y} E+B_{\xi} \mathrm{f}_{\xi} F\right) d y\right) \in \Omega^{2}(\mathcal{J}),
\end{aligned}
$$


так что класс эквивалентности $\bar{\omega} \in \mathrm{CL}^{1}(E F)$. Можно проверить, что $\bar{\omega}=0$ тогда и только тогда, когда $A=\lambda u_{x}, B=\lambda u_{y}, C=\lambda \mathrm{f}$ для некоторого $\lambda \in \mathbb{R}$. Далее,

$$
\begin{aligned}
0=d d \omega= & \left(\mathrm{D}_{t}\left(B_{\xi} F\right)-\mathrm{D}_{x}\left(B_{\xi} \mathrm{D}_{x} E+B_{\eta} \mathrm{D}_{y} E+B_{\xi} \mathrm{f}_{\xi} F\right)+\right. \\
& \left.+\mathrm{D}_{y}\left(A_{\xi} \mathrm{D}_{x} E+A_{\eta} \mathrm{D}_{y} E+A_{\eta} \mathrm{f}_{\eta} F\right)\right) d t \wedge d x \wedge d y= \\
= & P_{E}(\mathrm{D}) E+P_{F}(\mathrm{D}) F \in \Omega^{3}(\mathcal{E}),
\end{aligned}
$$

где

$$
\begin{aligned}
& P_{E}(\mathrm{D})=\left(-\mathrm{D}_{x} \circ\left(B_{\xi} \mathrm{D}_{x}+B_{\eta} \mathrm{D}_{y}\right)+\mathrm{D}_{y} \circ\left(A_{\xi} \mathrm{D}_{x}+A_{\eta} \mathrm{D}_{y}\right)\right) \otimes d t \wedge d x \wedge d y, \\
& P_{F}(\mathrm{D})=\left(\mathrm{D}_{t} \circ B_{\xi}-\mathrm{D}_{x} \circ B_{\xi} \mathrm{f}_{\xi}+\mathrm{D}_{y} \circ A_{\eta} \mathrm{f}_{\eta}\right) \otimes d t \wedge d x \wedge d y,
\end{aligned}
$$

так что $P(\mathrm{D})=\left\|P_{E}(\mathrm{D}), P_{F}(\mathrm{D})\right\| \in \Omega^{3}(\mathrm{DI})$ и $\overline{P(\mathrm{D})} \in H^{3}(\mathrm{DI})$.

4.3.3. Гиперболическая связь. Рассмотрим систему $E F=0$,

$$
E=u_{t}-\mathrm{f}(\xi, \eta), \quad F=u_{x x}-u_{y y}, \quad \xi=u_{x}, \eta=u_{y},
$$

где $\mathrm{f}_{\xi \xi}-\mathrm{f}_{\eta \eta}=0$. Пусть $A, B, C(\xi, \eta)$ - гладкие функции, удовлетворяющие уравнениям

$$
\left\{\begin{array} { l } 
{ A _ { \xi } = B _ { \eta } , } \\
{ A _ { \eta } = B _ { \xi } , }
\end{array} \quad \left\{\begin{array}{l}
C_{\xi}=A_{\xi} \mathrm{f}_{\xi}+A_{\eta} \mathrm{f}_{\eta}, \\
C_{\eta}=B_{\xi} \mathrm{f}_{\xi}+B_{\eta} \mathrm{f}_{\eta} .
\end{array}\right.\right.
$$

Рассмотрим форму $\omega=A d x+B d y+C d t \in \Omega^{1}(\mathcal{E})$. Дифференциал

$$
\begin{aligned}
d \omega= & \left(\mathrm{D}_{x} B-\mathrm{D}_{y} A\right) d x \wedge d y+d t \wedge\left(\left(\mathrm{D}_{t} A-\mathrm{D}_{x} C\right) d x+\left(\mathrm{D}_{t} B-\mathrm{D}_{y} C\right) d y\right)= \\
= & B_{\xi} F d x \wedge d y+d t \wedge\left(\left(A_{\xi} \mathrm{D}_{x} E+A_{\eta} \mathrm{D}_{y} E-A_{\eta} \mathrm{f}_{\eta} F\right) d x+\right. \\
& \left.+\left(B_{\xi} \mathrm{D}_{x} E+B_{\eta} \mathrm{D}_{y} E+B_{\xi} \mathrm{f}_{\xi} F\right) d y\right) \in \Omega^{2}(\mathcal{J}),
\end{aligned}
$$

так что класс эквивалентности $\bar{\omega} \in \mathrm{CL}^{1}(E F)$. Можно проверить, что $\bar{\omega}=0$ тогда и только тогда, когда $A=\lambda u_{x}, B=\lambda u_{y}, C=\lambda \mathrm{f}$ для некоторого $\lambda \in \mathbb{R}$. Далее,

$$
\begin{aligned}
0=d d \omega= & \left(\mathrm{D}_{t}\left(B_{\xi} F\right)-\mathrm{D}_{x}\left(B_{\xi} \mathrm{D}_{x} E+B_{\eta} \mathrm{D}_{y} E+B_{\xi} \mathrm{f}_{\xi} F\right)+\right. \\
& \left.+\mathrm{D}_{y}\left(A_{\xi} \mathrm{D}_{x} E+A_{\eta} \mathrm{D}_{y} E-A_{\eta} \mathrm{f}_{\eta} F\right)\right) d t \wedge d x \wedge d y= \\
= & P_{E}(\mathrm{D}) E+P_{F}(\mathrm{D}) F \in \Omega^{3}(\mathcal{E}),
\end{aligned}
$$

где

$$
\begin{aligned}
& P_{E}(\mathrm{D})=\left(-\mathrm{D}_{x} \circ\left(B_{\xi} \mathrm{D}_{x}+B_{\eta} \mathrm{D}_{y}\right)+\mathrm{D}_{y} \circ\left(A_{\xi} \mathrm{D}_{x}+A_{\eta} \mathrm{D}_{y}\right)\right) \otimes d t \wedge d x \wedge d y, \\
& P_{F}(\mathrm{D})=\left(\mathrm{D}_{t} \circ B_{\xi}-\mathrm{D}_{x} \circ B_{\xi} \mathrm{f}_{\xi}-\mathrm{D}_{y} \circ A_{\eta} \mathrm{f}_{\eta}\right) \otimes d t \wedge d x \wedge d y,
\end{aligned}
$$

так что $P(\mathrm{D})=\left\|P_{E}(\mathrm{D}), P_{F}(\mathrm{D})\right\| \in \Omega^{3}(\mathrm{DI})$ и $\overline{P(\mathrm{D})} \in H^{3}(\mathrm{DI})$.

4.4. Уравнения неразрывности. В некоторых приложениях система связей $F=0$ есть система уравнений неразрывности

$$
F=\left(F^{\kappa}\right) \in \mathcal{E}^{\mathrm{K}}, \quad F^{\kappa}=\partial_{x^{\mu}} u^{\kappa \mu} .
$$


Здесь пространства когомологий $H_{x}^{q}(\overline{\mathcal{E}})$ допускают полное описание. Именно, перепишем систему $\partial_{x^{\mu}} u^{\kappa \mu}=0$ как $\partial_{x^{1}} u^{\kappa 1}+\sum_{2 \leqslant \alpha \leqslant m} \partial_{x^{\alpha}} u^{\kappa \alpha}=0$ и поступим с ней как с эволюционной системой

$$
G=\partial_{s} v-\mathrm{g}=0, \quad v=\left(v^{\kappa}, v^{\kappa \alpha}\right) \in V, \quad \mathrm{~g}=\left(g^{k}, g_{\iota}^{\kappa \alpha}\right) \in \mathcal{F}^{A},
$$

где мы используем локальные обозначения:

- $s \in S=\mathbb{R}, y=\left(y^{2}, \ldots, y^{m}\right) \in Y=\mathbb{R}^{m-1}, s=x^{1}, y^{\alpha}=x^{\alpha}, 2 \leqslant \alpha \leqslant m$;

- $A=\mathrm{K} \cup B, B=\left\{(\kappa, \alpha, \iota) \mid \kappa \in \mathrm{K}, 2 \leqslant \alpha \leqslant m, \iota \in \mathbb{Z}_{+}\right\}$;

- $\mathbb{J}=\left\{j=\left(j^{\alpha}\right) \mid j^{\alpha} \in \mathbb{Z}_{+}, 2 \leqslant \alpha \leqslant m\right\}=\mathbb{Z}_{+}^{m-1}, \quad i=(\iota, j) \in \mathbb{I}$;

- $V=\left\{v=\left(v^{\kappa}, v_{\iota}^{\kappa \alpha}\right) \mid \kappa \in \mathrm{K},(\kappa, \alpha, \iota) \in B\right\}=\mathbb{R}^{A}$, $v^{\kappa}=v_{0}^{\kappa}=u^{\kappa 1}=u_{0}^{\kappa 1}, v_{\iota}^{\kappa \alpha}=v_{\iota 0}^{\kappa}=u_{(\iota, 0)}^{\kappa \alpha} ;$

- $\mathbf{V}=\left\{\mathbf{v}=\left(v_{j}^{\kappa}, v_{\iota j}^{\kappa \alpha}\right) \mid \kappa \in \mathrm{K},(\kappa, \alpha, \iota) \in B, j \in \mathbb{J}\right\}$, $v_{j}^{\kappa}=u_{(0, j)}^{\kappa 1}, v_{\iota j}^{\kappa \alpha}=u_{(\iota, j)}^{\kappa \alpha}$;

- $\mathcal{F}=\mathcal{C}_{\text {fin }}^{\infty}(S Y \mathbf{V} ; \mathbb{R})$;

- $D_{s}=\partial_{s}+\sum_{j \in \mathbb{J}}\left(g_{j}^{\kappa} \partial_{v_{j}^{\kappa}}+g_{\iota j}^{\kappa \beta} \partial_{v_{\iota j}^{\kappa \beta}}\right)$, $D_{\alpha}=\partial_{y^{\alpha}}+\sum_{j \in \mathbb{J}}\left(v_{j+(\alpha)}^{\kappa} \partial_{v_{j}^{\kappa}}+v_{\iota, j+(\alpha)}^{\kappa \beta} \partial_{v_{\iota j}^{\kappa \beta}}\right), 2 \leqslant \alpha \leqslant m$;

- $g^{\kappa}=-v_{0,(\beta)}^{\kappa \beta}, g_{\iota}^{\kappa \beta}=v_{\iota+1,0}^{\kappa \beta}, g_{j}^{\kappa}=D_{j} g^{\kappa}=-v_{0, j+(\beta)}^{\kappa \beta}, g_{\iota j}^{\kappa \beta}=D_{j} g_{\iota}^{\kappa \beta}=v_{\iota+1, j}^{\kappa \beta}$.

В силу теоремы 8 имеем

$$
H^{0}(\mathcal{F})=\mathbb{R}, \quad H^{q}(\mathcal{F})=0, \quad 1 \leqslant q \leqslant m-2,
$$

а согласно теореме 9

$$
H^{m-1}(\mathcal{F}) \simeq\left\{\chi \in \mathcal{F}_{A} \mid \chi_{*}=\chi^{*}, D_{s} \chi+\mathrm{g}^{*} \chi=0\right\}, \quad[\omega] \mapsto \chi=\delta_{v} J^{s},
$$

где $\omega=J^{s} d^{m-1} y+J^{\alpha} d s \wedge d_{\alpha} y, D_{s} J^{s}+D_{\alpha} J^{\alpha}=0$. Здесь,

$$
\mathrm{g}_{*}: \mathcal{F}^{A} \rightarrow \mathcal{F}^{A}, \quad \phi=\left(\phi^{\kappa}, \phi_{\iota}^{\kappa \alpha}\right) \mapsto \mathrm{g}_{*} \phi=\left(\left(\mathrm{g}_{*} \phi\right)^{\kappa},\left(\mathrm{g}_{*} \phi\right)_{\iota}^{\kappa \alpha}\right),
$$

где $\left(\mathrm{g}_{*} \phi\right)^{\kappa}=-D_{\beta} \phi_{0}^{\kappa \beta},\left(\mathrm{g}_{*} \phi\right)_{\iota}^{\kappa \alpha}=\phi_{\iota+1}^{\kappa \alpha}$, тогда как

$$
\mathrm{g}^{*}: \mathcal{F}_{A} \rightarrow \mathcal{F}_{A}, \quad \chi=\left(\chi_{\kappa}, \chi_{\kappa \alpha}^{\iota}\right) \mapsto \mathrm{g}^{*} \chi=\left(\left(\mathrm{g}^{*} \chi\right)_{\kappa},\left(\mathrm{g}^{*} \chi\right)_{\kappa \alpha}^{\iota}\right),
$$

где $\left(\mathrm{g}^{*} \chi\right)_{\kappa}=0,\left(\mathrm{~g}^{*} \chi\right)_{\kappa \alpha}^{\iota}=\chi_{\kappa \alpha}^{\iota-1}+\delta_{0}^{\iota} D_{\alpha} \chi_{\kappa}$ (заметим, что $\left.\chi_{\kappa \alpha}^{-1}=0\right)$. Далее, уравнение $D_{s} \chi+\mathrm{g}^{*} \chi=0$ сводится к системе

$$
D_{s} \chi=0, \quad D_{s} \chi_{\kappa \alpha}^{0}+D_{\alpha} \chi_{\kappa}=0, \quad D_{s} \chi_{\kappa \alpha}^{\iota}+\chi_{\kappa \alpha}^{\iota-1}=0
$$

и имеет общее решение $\chi=\left(\chi_{\kappa}, 0\right), \chi_{\kappa} \in \mathbb{R}, \kappa \in \mathrm{K}$ (решающий факт здесь состоит в том, что каждая функция $f \in \mathcal{F}$ имеет конечный порядок, поскольку зависит лишь от конечного числа аргументов; подробнее см., например, [3], [8], [12], [13]). Условие $\chi_{*}=\chi^{*}$ тривиально выполняется.

Мы возвращаемся к нашим глобальным обозначениям.

Tеорема 13. Система $F=0$, где $F=\left(F^{\kappa}\right) \in \mathcal{F}^{\mathrm{K}}, F^{\kappa}=\partial_{x^{\mu}} u^{\kappa \mu}, \kappa \in \mathrm{K}, \mathcal{F}=$ $\mathcal{C}^{\infty}(\mathrm{XU} ; \mathbb{R})$, имеет следующие пространства законов сохранения:

$$
\mathrm{CL}^{q}(F)= \begin{cases}0, & 0 \leqslant q \leqslant m-2, \\ \left\{\omega=\chi_{\kappa} e^{\kappa} \mid \chi_{\kappa} \in \mathbb{R}\right\} \simeq \mathbb{R}_{\mathrm{K}}, & q=m-1,\end{cases}
$$

где $e^{\kappa}=u^{\kappa \mu} d_{\mu} x$. 
ДокАЗАТЕЛЬСТво следует из предыдущих рассуждений и тривиального равенства $\delta_{v^{\kappa}} v^{\kappa^{\prime}}=\delta_{\kappa}^{\kappa^{\prime}}, \kappa, \kappa^{\prime} \in \mathrm{K}$.

ЗАмечание 1. Ясно, что здесь каждый ток вида $\omega=\chi_{\kappa} u^{\kappa \mu}, \chi_{\kappa} \in \mathbb{R}$, задает нетривиальный закон сохранения. Теорема 13 утверждает, что все законы сохранения имеют такой вид.

Следствие 2. Система связей $F=0$, где $F=\left(F^{\kappa}\right) \in \mathcal{E}^{\mathrm{K}}, F^{\kappa}=\partial_{x^{\mu}} u^{\kappa \mu}, \kappa \in \mathrm{K}$, $\mathcal{E}=\mathcal{C}^{\infty}(\mathrm{TXU} ; \mathbb{R})$, имеет следующие пространства когомологий:

$$
H_{x}^{q}(\overline{\mathcal{E}})= \begin{cases}\mathcal{T}, & q=0 \\ 0, & 1 \leqslant q \leqslant m-2, \\ \left\{\omega=\chi_{\kappa} e^{\kappa} \mid \chi_{\kappa} \in \mathcal{T}\right\} \simeq \mathcal{T}_{\mathrm{K}}, & q=m-1,\end{cases}
$$

где $\mathcal{T}=\mathcal{C}^{\infty}(\mathrm{T} ; \mathbb{R}), e^{\kappa}=u^{\kappa \mu} d_{\mu} x^{1)}$.

4.5. Связи в виде нулевой дивергенции. Эволюция со связью в виде нулевой дивергенции описывается системой $E F=0$, где

$$
E=\left(E^{\kappa \mu}=u_{t}^{\kappa \mu}-\mathrm{f}^{\kappa \mu}\right), \quad \mathrm{f}=\left(\mathrm{f}^{\kappa \mu}\right) \in \mathcal{E}^{\mathrm{K} \times m}, \quad F=\left(F^{\kappa}=\partial_{x^{\mu}} u^{\kappa \mu}\right) \in \mathcal{E}^{\mathrm{K}},
$$

так что здесь $\mathrm{A}=\mathrm{K} \times m, \mathbf{U}=\mathbb{R}_{\mathbb{I}}^{\mathrm{K} \times m}$. Мы предполагаем, что системы $E=0$ и $F=0$ совместные, т. е.

$$
D_{t} F^{\kappa}=D_{t} D_{\mu} u^{\kappa \mu}=D_{\mu} D_{t} u^{\kappa \mu}=D_{\mu} \mathrm{f}^{\kappa \mu} \in \mathcal{J}, \quad \kappa \in \mathrm{K} .
$$

В силу теоремы 12 и следствия 2 определены следующие точные последовательности:

$$
\begin{array}{ll}
0 \rightarrow H^{0}(\overline{\mathcal{E}}) \rightarrow \mathcal{T} \stackrel{\Delta}{\rightarrow} \mathcal{T}, & H^{0}(\overline{\mathcal{E}})=\left.\operatorname{Ker} \Delta\right|_{\mathcal{T}}, \\
\mathcal{T} \stackrel{\Delta}{\rightarrow} \mathcal{T} \rightarrow H^{1}(\overline{\mathcal{E}}) \rightarrow 0, & H^{1}(\overline{\mathcal{E}})=\mathcal{T} /\left.\operatorname{Im} \Delta\right|_{\mathcal{T}}, \\
0 \rightarrow H^{q}(\overline{\mathcal{E}}) \rightarrow 0, & H^{q}(\overline{\mathcal{E}})=0, \quad 2 \leqslant q \leqslant m-2, \\
0 \rightarrow H^{m-1}(\overline{\mathcal{E}}) \stackrel{\bar{\pi}_{x}}{\longrightarrow} H_{x}^{m-1}(\overline{\mathcal{E}}) \stackrel{\Delta}{\rightarrow} H_{x}^{m-1}(\overline{\mathcal{E}}), & H^{m-1}(\overline{\mathcal{E}})=\operatorname{Ker} \Delta^{m-1},
\end{array}
$$

где $\Delta^{m-1}=\left.\Delta\right|_{H_{x}^{m-1}(\overline{\mathcal{E}})}: H_{x}^{m-1}(\overline{\mathcal{E}}) \rightarrow H_{x}^{m-1}(\overline{\mathcal{E}}), \Delta^{m-1}[\omega]=\left[D_{t} \omega\right]$. Легко проверяется, что

$$
\begin{aligned}
& \left.\operatorname{Ker} \Delta\right|_{\mathcal{T}}=\mathbb{R},\left.\quad \operatorname{Im} \Delta\right|_{\mathcal{T}}=\mathcal{T}, \\
& \operatorname{Ker} \Delta^{m-1}=\left\{\omega=\chi_{\kappa} e^{\kappa} \mid \chi_{\kappa} \in \mathcal{T}, \partial_{t} \chi_{\kappa}+\chi_{\sigma} L_{\kappa}^{\sigma}=0\right\} \simeq \mathbb{R}_{K},
\end{aligned}
$$

где в силу условия совместности

$$
\left[D_{t} e^{\sigma}\right]=\left[\left(D_{t} u^{\sigma \mu}\right) d_{\mu} x\right]=\left[\mathrm{f}^{\sigma \mu} d_{\mu} x\right]=L_{\kappa}^{\sigma} e^{\kappa}, \quad L_{\kappa}^{\sigma}=\delta_{u^{\kappa 1}} \mathrm{f}^{\sigma 1} \in \mathcal{T} .
$$

Таким образом, справедлива следующая теорема.

\footnotetext{
1) Отметим, что здесь переменная $t \in \mathrm{T}$ является параметром.
} 
Теорема 14. Пространства законов сохранения приведенной выше системы $E F=0$ имеют вид

$$
\mathrm{CL}^{q}(E F)= \begin{cases}0, & 0 \leqslant q \leqslant m-2, \\ \left\{\omega=\chi_{\kappa} e^{\kappa} \mid \chi_{\kappa} \in \mathcal{T}, \partial_{t} \chi_{\kappa}+\chi_{\sigma} L_{\kappa}^{\sigma}=0\right\} \simeq \mathbb{R}_{\mathrm{K}}, & q=m-1 .\end{cases}
$$

ЗАмечАниЕ 2. Пространство $\mathrm{CL}^{m-1}(E F)$ имеет другое (более естественное) представление. Именно, согласно равенству $H^{m-1}(\overline{\mathcal{E}})=\operatorname{Ker} \Delta^{m-1}$ у каждой базисной формы $e^{\kappa} \in \operatorname{Ker} \Delta^{m-1}, \kappa \in \mathrm{K}$, имеется базисный класс эквивалентности $\left[J^{\kappa}\right] \in$ $H^{m-1}(\overline{\mathcal{E}})$ такой, что $\bar{\pi}_{x}\left[J^{\kappa}\right]=e^{\kappa}$, где $J^{\kappa}=e^{\kappa}+d t \wedge \psi^{\kappa} \in \Omega^{m-1}(\mathcal{E}), d J^{\kappa} \in \Omega^{m}(\mathcal{J})$, $\psi^{\kappa} \in \Omega_{x}^{m-2}(\mathcal{E}), d_{x} \psi^{\kappa}-D_{t} e^{\kappa} \in \Omega^{m-1}(\mathcal{J})$. Следовательно,

$$
\mathrm{CL}^{m-1}(E F)=\left\{\chi_{\kappa}\left[J^{\kappa}\right] \mid \partial_{t} \chi_{\kappa}+\chi_{\sigma} L_{\kappa}^{\sigma}=0\right\} .
$$

ЗАМЕЧАниЕ 3. Эволюционные системы с управляемой дивергенцией

$$
E^{\kappa \mu}=u_{t}^{\kappa \mu}-\mathrm{f}^{\kappa \mu}=0, \quad F^{\kappa}=\partial_{x^{\mu}} u^{\kappa \mu}-\rho^{\kappa}=0,
$$

где $\rho^{\kappa} \in \mathcal{C}^{\infty}(\mathrm{TX} ; \mathbb{R}) \subset \mathcal{E}$, сводятся к системам с нулевой дивергенцией

$$
\tilde{E}^{\kappa \mu}=v_{t}^{\kappa \mu}-\mathrm{g}^{\kappa \mu}=0, \quad \tilde{F}^{\kappa}=\partial_{x^{\mu}} v^{\kappa \mu}=0
$$

заменой переменных

$$
v^{\kappa \mu}=u^{\kappa \mu}-\varphi^{\kappa \mu}, \quad \mathrm{g}^{\kappa \mu}(t, x, \mathbf{v})=\mathrm{f}^{\kappa \mu}(t, x, \mathbf{u})-\partial_{t} \varphi^{\kappa \mu},
$$

где $\varphi(t, x)$ - частное решение системы $F=0$, т. е. $\partial_{x^{\mu}} \varphi^{\kappa \mu}-\rho^{\kappa}=0$.

4.6. Пример: уравнения Максвелла. В стандартных физических обозначениях

- $t \in \mathrm{T}=\mathbb{R}, x \in \mathrm{X}=\mathbb{R}^{3}$, - временна́я и пространственные переменные,

- $\mathbf{E}, \mathbf{H} \in \mathrm{V}=\mathbb{R}^{3}$ - электрическое и магнитное поля,

- $\rho(t, x), \mathbf{j}(t, x)$ - заданные плотности заряда и тока

уравнения Максвелла имеют вид

$$
\begin{array}{ll}
\partial_{t} \mathbf{H}+\operatorname{rot} \mathbf{E}=0, & \operatorname{div} \mathbf{H}=0, \\
\partial_{t} \mathbf{E}-\operatorname{rot} \mathbf{H}+\mathbf{j}=0, & \operatorname{div} \mathbf{E}-\rho=0 .
\end{array}
$$

Условие совместности здесь имеет вид

$$
\partial_{t} \rho+\operatorname{div} \mathbf{j}=0
$$

и приводит к представлению $\rho=\operatorname{div} \mathbf{R}, \mathbf{j}=-\partial_{t} \mathbf{R}+\operatorname{rot} \mathbf{S}$ с некоторыми векторными функциями $\mathbf{R}(t, x)$ и $\mathbf{S}(t, x)$. Это позволяет переписать уравнения Максвелла в виде системы с нулевой дивергенцией:

$$
\begin{array}{ll}
\partial_{t} \mathbf{H}+\operatorname{rot} \mathbf{E}=0, & \operatorname{div} \mathbf{H}=0, \\
\partial_{t}(\mathbf{E}-\mathbf{R})-\operatorname{rot}(\mathbf{H}-\mathbf{S})=0, & \operatorname{div}(\mathbf{E}-\mathbf{R})=0 .
\end{array}
$$


Итак, здесь $\mathrm{K}=\{1,2\}, m=3$,

$$
\begin{array}{ll}
u^{1 \lambda}=H^{\lambda}, & u^{2 \lambda}=E^{\lambda}-R^{\lambda}, \quad 1 \leqslant \lambda \leqslant 3, \\
\mathrm{f}^{1 \lambda}=-\operatorname{rot} \mathbf{H}^{\lambda}=-e^{\lambda \mu \nu} \partial_{x^{\mu}} H_{\nu}, & \mathrm{f}^{2 \lambda}=\operatorname{rot}(\mathbf{E}-\mathbf{R})^{\lambda}=e^{\lambda \mu \nu} \partial_{x^{\mu}}\left(E_{\nu}-R_{\nu}\right), \\
F^{1}=\operatorname{div} \mathbf{H}=\partial_{x^{\mu}} H^{\mu}, & F^{2}=\operatorname{div}(\mathbf{E}-\mathbf{R})=\partial_{x^{\mu}}\left(E^{\mu}-R^{\mu}\right)
\end{array}
$$

(в евклидовом пространстве компоненты $V_{\mu}=\delta_{\mu \nu} V^{\nu}=V^{\mu}$ для любого вектора $\mathbf{V}$ ). Далее,

$$
\begin{array}{ll}
e^{1}=H^{\mu} d_{\mu} x, & d_{x} e^{1}=d_{x} H^{\mu} d_{\mu} x=(\operatorname{div} \mathbf{H}) d^{m} x \in \Omega_{x}^{m}(\mathcal{J}), \\
e^{2}=\left(E^{\mu}-R^{\mu}\right) d_{\mu} x, & d_{x} e^{2}=d_{x}\left(E^{\mu}-R^{\mu}\right) d_{\mu} x=\operatorname{div}(\mathbf{E}-\mathbf{R}) d^{m} x \in \Omega_{x}^{m}(\mathcal{J}) .
\end{array}
$$

Так как $\delta_{u^{\kappa 1}} \mathrm{f}^{\sigma 1}=0, \kappa, \sigma \in \mathrm{K}$, то $L_{\kappa}^{\sigma}=0$, и система $\partial_{t} \chi_{\kappa}+\chi_{\sigma} L_{\kappa}^{\sigma}=0$ имеет общее решение $\chi_{\kappa} \in \mathbb{R}$.

Наконец, можно положить

$$
J^{1}=H^{\mu} d_{\mu} x-d t \wedge E_{\mu} d x^{\mu}, \quad J^{2}=\left(E^{\mu}-R^{\mu}\right) d_{\mu} x+d t \wedge\left(H_{\mu}-S_{\mu}\right) d x^{\mu},
$$

поскольку в этом случае $\bar{\pi}_{x} J^{\kappa}=e^{\kappa}$ для всех $\kappa \in \mathrm{K}$, и

$$
\begin{aligned}
& d J^{1}=(\operatorname{div} \mathbf{H}) d^{m} x+d t \wedge\left(\partial_{t} \mathbf{H}+\operatorname{rot} \mathbf{E}\right)^{\mu} d_{\mu} x=0, \\
& d J^{2}=(\operatorname{div}(\mathbf{E}-\mathbf{R})) d^{m} x+d t \wedge\left(\partial_{t}(\mathbf{E}-\mathbf{R})-\operatorname{rot}(\mathbf{H}-\mathbf{S})\right)^{\mu} d_{\mu} x=0
\end{aligned}
$$

на решениях уравнений Максвелла. Итак, согласно приведенным рассуждениям справедливо следующее представление:

$$
\mathrm{CL}^{m-1}(E F)=\left\{\chi_{\kappa} e^{\kappa} \mid \chi_{\kappa} \in \mathbb{R}\right\}=\left\{\chi_{\kappa} J^{\kappa} \mid \chi_{\kappa} \in \mathbb{R}\right\} \simeq \mathbb{R}_{2} .
$$

Уравнения Максвелла имеют красивое описание на языке дифференциальных форм. Именно, тензор электромагнитного поля $F$ записывается как 2-форма

$$
F=H^{\mu} d_{\mu} x-E_{\mu} d t \wedge d x^{\mu}=J^{1}
$$

его сопряженный по Ходжу тензор *F есть 2-форма

$$
* F=E^{\mu} d_{\mu} x+d t \wedge H_{\mu} d x^{\mu}=J^{2}+\left(R^{\mu} d_{\mu} x+S_{\mu} d t \wedge d x^{\mu}\right),
$$

а уравнения Максвелла записываются в виде (см., например, [14])

$$
d F=d J^{1}=0, \quad d(* F)-\left(\rho d^{3} x-j^{\mu} d t \wedge d_{\mu} x\right)=d J^{2}=0 .
$$

Заметим, что

$$
\left.d\left(R^{\mu} d_{\mu} x+S_{\mu} d t \wedge d^{\mu}\right)=\rho d^{3} x-j^{\mu} d t \wedge d_{\mu} x\right) .
$$

Таким образом, в этом подходе уравнения Максвелла записываются как двумерные законы сохранения. В силу теоремы 14 они не допускают каких-либо других маломерных законов сохранения. 


\section{Список литературы}

[1] В. В. Жаринов, ТМФ, 68:2 (1986), 163-171.

[2] В. В. Жаринов, Матем. сб., 181:11 (1990), 1475-1485.

[3] V. V. Zharinov, Lecture Notes on Geometrical Aspects of Partial Differential Equations, Series on Soviet and East European Mathematics, 9, World Sci., Singapore, 1992.

[4] В. В. Жаринов, Матем. сб., 184:5 (1993), 39-54.

[5] V. V. Zharinov, Тр. МИАН, 203 (1994), 478-493.

[6] В. В. Жаринов, ТМФ, 163:1 (2010), 3-16.

[7] В. В. Жаринов, ТМФ, 174:2 (2013), 256-271.

[8] П. Олвер, Приложения групп Ли к дифференциальным уравнениям, Мир, М., 1989.

[9] E. R. Kolchin, Differential Algebra and Algebraic Groups, Academic Press, New York, 1973.

[10] J. F. Ritt, Differential Algebra, Dover, New York, 1966.

[11] С. Маклейн, Гомология, Мир, М., 1966.

[12] T. Tsujishita, Osaka J. Math., 19:2 (1982), 311-363.

[13] А. М. Виноградов, И. С. Красильщик, В. В. Лычагин, Введение в геометрию нелинейных дифференииальных уравнений, Наука, М., 1986.

[14] Р. Ботт, Л. В. Ту, Дифференииальные формы в алгебраической топологии, Наука, М., 1989.

Поступила в редакцию 5.05.2015 